\title{
Dual finite-element analysis using second-order cone programming for structures including contact
}

\author{
Chadi El Boustani ${ }^{\mathrm{a}, \mathrm{b}, *}$, Jeremy Bleyer, ${ }^{\mathrm{a}, *}$, Mathieu Arquier ${ }^{\mathrm{b}}$, Mohammed-Khalil Ferradi ${ }^{\mathrm{b}}$, \\ Karam $\mathrm{Sab}^{\mathrm{a}}$ \\ ${ }^{a}$ Laboratoire Navier UMR 8205 (ENPC-IFSTTAR-CNRS), Université Paris-Est, 6-8 av Blaise Pascal, \\ 77455 Marne-la-Vallée, FRANCE \\ ${ }^{b}$ Strains, 23 avenue d'Italie, 75013 Paris, FRANCE
}

\begin{abstract}
Computation of elastic structures in contact is performed by means of a dual analysis combining displacement-based and equilibrium-based finite elements. Contact conditions are formulated in the framework of second-order cone programmaing (SOCP) and an efficient interior point method (IPM) algorithm is presented for the resolution of the associated optimization problems. The dual approach allows the user to assess the quality of convergence and to efficiently calculate a discretization error estimator which includes a contact error term. An efficient remeshing scheme, based on the local contributions of the elements to the global error, can then be used to efficiently improve the solution accuracy. The whole process is illustrated on some examples and applied to a typical steel assembly. Its efficiency, in particular concerning the IPM solver, is demonstrated in comparison with the industrial finite element code Abaqus.
\end{abstract}

Keywords: contact elastostatics, equilibrium finite elements, error estimator, second order cone programing, interior point method

\section{Introduction}

The study of large scale contact problems between elastic bodies has always been a main interest of mechanical and structural engineers. Though the finite element method is well established for simple 3D elastic continuum, the treatment of contact conditions between bodies remains a problematic matter for inexperienced users. Contact between mechanical parts exists in almost all engineering fields, specifically in structural analysis where the verification of steel assemblies relies on determining the forces and displacements in the assembly which, by definition, joins distinct parts of the structure, transferring forces

\footnotetext{
${ }^{*}$ Corresponding author

Email addresses: chadi.el-boustani@enpc.fr (Chadi El Boustani), jeremy.bleyer@enpc.fr (Jeremy Bleyer), mathieu.arquier@strains.fr (Mathieu Arquier), mohammed-khalil.ferradi@strains.fr (Mohammed-Khalil Ferradi), karam.sab@enpc.fr (Karam $\mathrm{Sab})$
} 
through welds and bolts that mainly rely on frictional contact. Such situations present a major difficulty for structural engineers as the modelling of a complex steel assembly requires a great amount of computer-aided design time, whereas convergence of numerical solvers may often be challenging to achieve for inexperienced users.

Resolution of finite-elements computations including strong material non-linearities such as plasticity or contact therefore requires some expertise when aiming at obtaining accurate estimates of quantities of interest such as contact pressures or gaps, while trying to keep computational costs at a moderate level. In the present paper, we propose an approach for computing structures with contact such as 3D steel assemblies by using a solution algorithm which exhibits very robust convergence properties and does not require any fine-tuning algorithmic parameters while still being very competitive in terms of computational costs compared to classical approaches implemented in commercial software.

As for computational methods, contact problems are often solved using the penalty approach in which penetration between the two bodies, i.e. negative gap and tensile surface tractions are permitted, but penalized using a gap-element characterized by a large stiffness value [1]. This method is easy to implement and transforms the problem into a regularized unconstrained minimization problem, the drawback being that the system becomes ill-conditioned for increasingly larger penalty factors. Besides, contact constraints violation generally produces poor results, underestimating the system's stresses, and thus preventing engineers from performing a safe and optimized design. Another method consists in enforcing the contact conditions using Lagrange multipliers thus transforming the problem into a saddle point problem with simple inequality constraints [1]. Although contact conditions will be strictly verified, this method proved slow convergence rates. Augmented Lagrangian (AL) approaches emerged as an alternative [2, 3]: it couples the Lagrange multipliers approach with the penalty approach while limiting the need to drive the stiffness coefficient to infinity, thereby providing better results [4, 1, 5].

Strategies for solving the corresponding non-linear problem are numerous, including fixed-point scheme or Uzawa algorithm [6, 7], generalized/semi-smooth Newton methods [8, 9], primal-dual active sets [10], etc. Some of these methods have been shown to be equivalent in some specific cases [11, 12]. Frictional contact can be written as secondorder cone complementarity conditions [13, 14, 15] but the non-associated character of the Coulomb friction law makes the problem even more challenging, the problem becoming a non-monotone complementarity problem. Linear complementarity solvers have been proposed when adopting a facetized representation of the Coulomb cone [16, 17]. Bipotential approaches have also been studied along with dedicated numerical procedures [18, 19].

In the present work, we will consider the case of associated friction law, departing from the classic Coulomb friction. In such a situation, the complementarity problem becomes a convex second-order cone problem for which very efficient interior-point algorithms have been developed by the mathematical programming community [20, 21, 22, 23]. It is interesting to note that interior-point methods (IPM) are becoming a state-of-the art numerical procedure for other non-smooth mechanical problems such as limit load computations in limit analysis [24, 25, 26, 27] or computation of viscoplastic fluid flows for which conven- 
tional methods such as AL approaches have quite slow convergence rates [28, 29]. Only few attempts were made to solve contact problems using IPMs such as [30, 31] for modelling granular contact dynamics. While, earlier attempts [32, 33] did not use the most efficient forms of IPM methods, more recent implementations showed promising results in non-smooth contact dynamics [34] or isogeometric [35] contexts.

As regards our choice of considering only associated friction for the present paper, we point out that IPM rely on a convex conic optimization setting which does not hold in the presence of classical Coulomb friction. However, since quasi-static computations are considered here, we expect that the obtained solutions will be quite similar to a classical Coulomb friction computation, as it will be later shown in the example section. Moreover, some iterative strategies have already been proposed to tackle the case of non-associative plasticity or friction using a series of associated computations [36, 37]. The associated formulation of the IPM problems will therefore serve as a basis of a future extension to non-associated behaviours.

In this paper, we aim at further establishing the potential of IPM for contact of complex 3D assemblies, especially concerning its convergence robustness and low iteration count in regards of its use by inexperienced engineer. The proposed methodology can handle large scale problems and complex assemblies in an efficient manner with a minimum user intervention. We will also combine it with dual stress and displacement-based computations to assess the solution quality with respect to discretization errors. In summary, the present paper will include:

- the reformulation of associated frictional contact conditions as a pair of complementary dual cones to be used within the framework of second order cone programming (SOCP) (section 2);

- the reformulation of the minimization problems and an error estimator including a surface contact term relying on displacement and stress-based variational bounding principles (section 3);

- finite elements discretization for each of the two approaches based on classic kinematic elements [38] and on improved equilibrium elements [39, 40, 41, 42] (section 44;

- an adaptation of the IPM to take into account a quadratic objective function over conic conditions with state-of-the-art improvements such as Mehrotra predictorcorrector scheme [43] or self scaled cones [44] (section 5.1);

- the use of the error estimator in a global 3D remeshing scheme based on the dual approach used in this paper (section 5.2).

The process of solving the minimization problems, calculating errors and remeshing, is automatized and included in Strains' software ${ }^{1}$ "DS-Steel" which specialises in steel

\footnotetext{
${ }^{1}$ https://strains.fr/
} 
assemblies calculations ${ }^{2}$. The numerical examples presented in this paper (section 6) are produced using this process and by setting a target convergence value which is defined using the dual approach. The results are then compared with respect to computations made using Abaqus [45] to assess computational costs and the quality of results.

\section{Reference problem and governing equations}

\subsection{Contact constitutive equations}

Let us consider in this section the formulation of contact laws on the contact boundary denoted by $\Gamma_{\boldsymbol{c}}$ described by a unit normal vector $\boldsymbol{n}$ pointing from the solid $\Omega_{1}$ to the solid $\Omega_{2}$, and two tangential vectors $\boldsymbol{t}_{1}, \boldsymbol{t}_{2}$ (see figure 1). We will also use the following notation $\boldsymbol{x}=x_{N} \boldsymbol{n}+\boldsymbol{x}_{T}$ with $\boldsymbol{x}_{T}$ the tangential component of any vector $\boldsymbol{x}$.

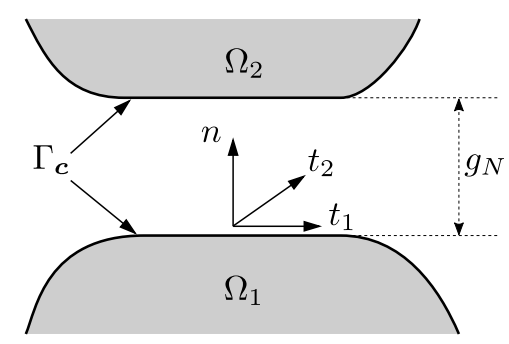

Figure 1: Local coordinate system

In the case where the two deformable bodies $\Omega_{1}$ and $\Omega_{2}$ come in contact, kinematic variables will involve the displacement discontinuity vector $\llbracket \boldsymbol{u} \rrbracket=\boldsymbol{u}_{2}-\boldsymbol{u}_{1}$, while the stress vector $\boldsymbol{T}=\sigma_{N} \boldsymbol{n}+\boldsymbol{\sigma}_{T}$ is expressed on one of the two faces since equilibrium conditions ensures equality between the two surfaces tractions on both sides of $\Gamma_{\boldsymbol{c}}$. Since the faces are not necessarily initially in contact, an initial gap vector $\boldsymbol{g}_{0}=g_{0} \boldsymbol{n}$ is introduced, the initial gap having no tangential component while its normal component is positive $g_{0} \geq 0$. This choice is appropriate considering that we will restrict to infinitesimal transformations and since contact will be implemented in a node-to-node fashion. As a consequence, meshes of both contacting surfaces will be conforming. The current gap vector is then defined by:

$$
\boldsymbol{g}_{(\boldsymbol{u})}=\left(\begin{array}{c}
g_{N} \\
\boldsymbol{g}_{T}
\end{array}\right)=\left(\begin{array}{c}
g_{0}+\llbracket u_{N} \rrbracket \\
\llbracket \boldsymbol{u}_{T} \rrbracket
\end{array}\right)
$$

\subsubsection{Unilateral contact}

Unilateral contact is enforced using Signorini's conditions which express the non-penetration and the non-apparition of tensile surface tractions between contact surfaces and the com-

\footnotetext{
$\sqrt[2]{\text { https://digital-structure.com/ }}$
} 
plementarity between normal stresses and the normal gap:

$$
\left\{\begin{array}{l}
g_{N} \geq 0 \\
\sigma_{N} \leq 0 \\
g_{N} \cdot \sigma_{N}=0
\end{array}\right.
$$

\subsubsection{Frictional behaviour}

Coulomb frictional model is used to model tangential behaviour of contact surfaces by means of the friction coefficient $\mu$. One distinguishes between stick and slip conditions (expressed here in terms of a quasistatic displacement increment):

$$
\left\{\begin{array}{l}
\text { if }\left\|\boldsymbol{\sigma}_{T}\right\|<-\mu \sigma_{N} \text { then } \boldsymbol{g}_{T}=0 \\
\text { if }\left\|\boldsymbol{\sigma}_{T}\right\|=-\mu \sigma_{N} \text { then } \boldsymbol{g}_{T}=\lambda \frac{\boldsymbol{\sigma}_{T}}{\left\|\boldsymbol{\sigma}_{T}\right\|} \text { with } \lambda \geq 0
\end{array}\right.
$$

This law shows the dependency over a frictional yield value $\mu \sigma_{N}$ and expresses the tangential slip as proportional to frictional stresses.

\subsubsection{Contact complementarity conditions}

When associated contact is assumed, the unilateral and frictional laws are both replaced by the following combined law:

$$
\left\{\begin{array}{l}
\text { if }\left\|\boldsymbol{\sigma}_{T}\right\|<-\mu \sigma_{N} \text { then } \boldsymbol{g}=\mathbf{0} \\
\text { if }\left\|\boldsymbol{\sigma}_{T}\right\|=-\mu \sigma_{N} \text { then } \boldsymbol{g}_{T}=\lambda \frac{\boldsymbol{\sigma}_{T}}{\left\|\boldsymbol{\sigma}_{T}\right\|} \text { with } \lambda=\frac{g_{N}}{\mu}
\end{array}\right.
$$

which ensures that $\sigma_{N} \leq 0, g_{N} \geq 0$ but also couples the normal and tangential gaps. The previous condition can be expressed as the following two second-order cones (see figure 2):

$$
\begin{gathered}
\left(\sigma_{N}, \boldsymbol{\sigma}_{T}\right) \in \mathcal{K}_{\mu} \\
\left(g_{N}, \boldsymbol{g}_{T}\right) \in \mathcal{K}_{-1 / \mu} \\
\text { where } K_{\alpha}=\left\{(x, \boldsymbol{y}) \in \mathbb{R} \times \mathbb{R}^{2} \mid\|\boldsymbol{y}\|+\alpha x \leq 0\right\}
\end{gathered}
$$

along with the following complementarity condition:

$$
\sigma_{N} g_{N}+\boldsymbol{\sigma}_{T} \cdot \boldsymbol{g}_{T}=0
$$

In the above, it can be observed that the cone $\mathcal{K}_{-1 / \mu}=\left(\mathcal{K}_{\mu}\right)^{\circ}=\mathcal{K}_{\mu}^{\circ}$ where $\star^{\circ}$ refers to the the polar cone. The pair $\left(\sigma_{N}, \boldsymbol{\sigma}_{T}\right)$ and $\left(g_{N}, \boldsymbol{g}_{T}\right)$ therefore satisfies a complementarity condition over dual cones. This remark is at the origin of the formulation of contact problems as complementarity problems in the general (non-associated) case and secondorder cone programming in the present associated case. Let us remark that the use of 
associated friction implies that slip only occurs when there is a normal opening. This aspect is indeed less realistic than a non-associated framework. However, the use of associated friction is interesting in the context of convex optimization solvers and can serve as a basis for iterative procedures when tackling the non-associated case which will be investigated in future works, see more details in [46, 14]. Illustrative applications of section 6 will also assess the difference between both models.
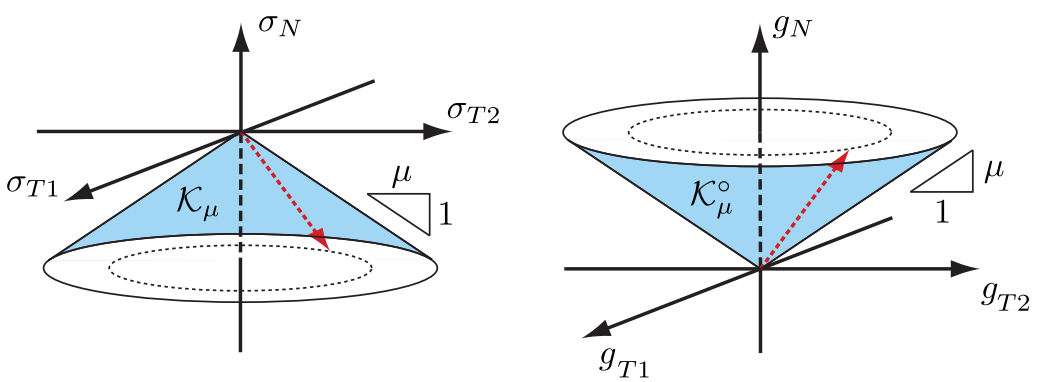

Figure 2: Geometry of the friction cones

\subsection{Local governing equations}

In the following, $\boldsymbol{u}$ denotes the displacement field, $\boldsymbol{\varepsilon}_{(\boldsymbol{u})}=\boldsymbol{\nabla}^{S} \boldsymbol{u}$ the linearized strain tensor, $\boldsymbol{\sigma}$ the Cauchy stress tensor and $\mathbb{C}$ the elastic stiffness tensor. Imposed displacements and surface tractions will be respectively denoted by $\boldsymbol{u}_{d}$ and $\boldsymbol{t}_{d}$, and the body forces by $\boldsymbol{b}$. The normal vector $\boldsymbol{n}$ pointing outwards will be used to orient free faces and surfaces. For contact conditions, the direction of the normal vector will be specified when needed.

Let $\Omega=\Omega_{1} \cup \Omega_{2}$ be the total space occupied by the solids sharing a potential contact surface $\Gamma_{c}$ and let be $\Sigma_{\boldsymbol{\sigma}}$ an internal surface of potential stress discontinuities (see figure 3). The external boundary will be split into three distinct parts such as: $\Gamma=\Gamma_{\boldsymbol{u}} \cup \Gamma_{\boldsymbol{t}} \cup \Gamma_{\boldsymbol{c}}$ and $\Gamma_{\boldsymbol{u}} \cap \Gamma_{\boldsymbol{t}} \cap \Gamma_{\boldsymbol{c}}=\emptyset$ where:

- displacements $\boldsymbol{u}$ are imposed to $\boldsymbol{u}_{d}$ on $\Gamma_{\boldsymbol{u}}=\Gamma_{1, \boldsymbol{u}} \cup \Gamma_{2, \boldsymbol{u}}$;

- surface tractions $\boldsymbol{t}=\boldsymbol{\sigma} \cdot \boldsymbol{n}$ are imposed to $\boldsymbol{t}_{d}$ on $\Gamma_{\boldsymbol{t}}=\Gamma_{1, t} \cup \Gamma_{2, \boldsymbol{t}}$;

- contact conditions between $\Omega_{1}$ and $\Omega_{2}$ are imposed on $\Gamma_{c}$. Generally the contact surface is unknown but in the scope of this article and the small displacements hypothesis, it is considered that the contact surface is known, predefined and does not change.

The general governing equations for an elastic body are the following:

\section{Equilibrium and static boundary conditions}

$$
\begin{array}{cc}
\operatorname{Div}(\boldsymbol{\sigma})+\boldsymbol{b}=0 & \text { in } \Omega \\
\llbracket \boldsymbol{\sigma} \rrbracket \cdot \boldsymbol{n}=0 & \text { on } \Sigma_{\boldsymbol{\sigma}} \\
\boldsymbol{\sigma} \cdot \boldsymbol{n}=\boldsymbol{t}_{d} & \text { on } \Gamma_{\boldsymbol{t}}
\end{array}
$$




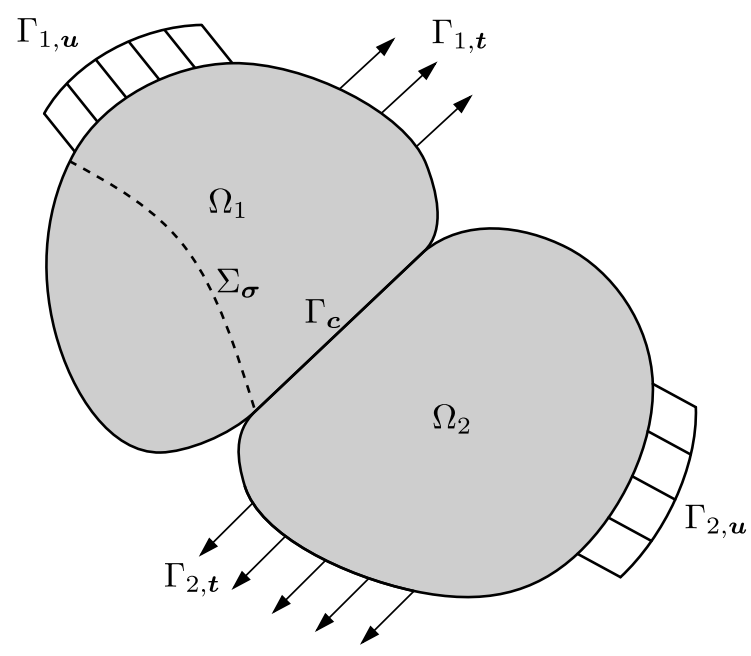

Figure 3: Reference model

Strain-displacement compatibility and kinematic boundary conditions

$$
\begin{gathered}
\boldsymbol{\varepsilon}_{(\boldsymbol{u})}=\nabla^{S} \boldsymbol{u} \quad \text { in } \Omega \\
\boldsymbol{u}=\boldsymbol{u}_{d} \quad \text { on } \Gamma_{\boldsymbol{u}}
\end{gathered}
$$

Elastic constitutive law

$$
\boldsymbol{\sigma}=\mathbb{C}: \varepsilon_{(u)} \quad \text { in } \Omega
$$

Contact constitutive laws between $\boldsymbol{\sigma} . \boldsymbol{n}$ and $\boldsymbol{g}_{(u)}$ on $\Gamma_{\boldsymbol{c}}$ as discussed in section 2.1.

\subsubsection{Statically admissible stress field}

A stress field $\boldsymbol{\sigma} \in \mathbb{S}$ belonging to the set $\mathbb{S}$ of symmetric second-rank tensors is said to be statically admissible (SA) with the body forces $\boldsymbol{b}$ in $\Omega$ and the imposed surfaces tractions $\boldsymbol{t}_{d}$ on $\Gamma_{t}$ if it satisfies the equilibrium equations with these data:

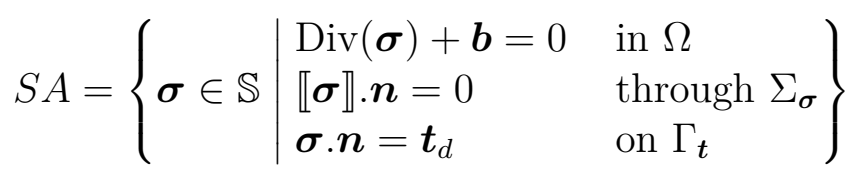

\subsubsection{Kinematically admissible displacement field}

A displacement field $\boldsymbol{u}: \Omega \rightarrow \mathbb{R}^{3}$ is said to be kinematically admissible (KA) with the imposed displacements $\boldsymbol{u}_{d}$ on $\Gamma_{\boldsymbol{u}}$ if it is piecewise continuous and continuously differentiable and such that $\boldsymbol{u}=\boldsymbol{u}_{d}$ on $\Gamma_{\boldsymbol{u}}$ :

$$
K A=\left\{\boldsymbol{u} \in \mathbb{R}^{3} \mid \boldsymbol{u}=\boldsymbol{u}_{d} \quad \text { on } \Gamma_{\boldsymbol{u}}\right\}
$$




\section{Primal and dual variational principles}

The following variational principles can be derived using the constitutive error estimator presented in appendix A:

$$
e_{(\boldsymbol{u}, \boldsymbol{\sigma})}^{2}=e_{\Omega(\boldsymbol{u}, \boldsymbol{\sigma})}^{2}+e_{\Gamma_{\boldsymbol{c}}(\boldsymbol{u}, \boldsymbol{\sigma})}^{2}
$$

Finding an approximate solution consists of computing an optimal pair $\left(\boldsymbol{u}_{h}, \boldsymbol{\sigma}_{h}\right)$ of displacements and stress fields such that the estimated error, and thus the real error, are minimal over subsets of the space of K.A. displacements and S.A. stress fields, respectively noted $K A_{h} \subseteq K A$ and $S A_{h} \subseteq S A$ :

$$
\begin{aligned}
\left(\boldsymbol{u}_{h}, \boldsymbol{\sigma}_{h}\right)=\underset{\boldsymbol{u}, \boldsymbol{\sigma}}{\arg \min } & e_{(\boldsymbol{u}, \boldsymbol{\sigma})}^{2} \\
\text { subject to } & (\boldsymbol{u}, \boldsymbol{\sigma}) \in K A_{h} \times S A_{h}
\end{aligned}
$$

Both functionals $\Pi_{p}$ and $\Pi_{c}$ are a sum of a positive definite quadratic function, some linear terms and indicator functions of convex sets thus they are strictly convex. Since the error functional is the sum of two functions of $\boldsymbol{u}$ and $\boldsymbol{\sigma}$ respectively, the above problem can be transformed into two variational forms, namely a kinematic approach in which a kinematically admissible field is obtained by minimizing the total potential energy (TPE) $\Pi_{p}$ :

$$
\begin{array}{lc}
\min _{\boldsymbol{u}} & \Pi_{p(\boldsymbol{u})}=\int_{\Omega} \frac{1}{2} \boldsymbol{\varepsilon}_{(\boldsymbol{u})}: \mathbb{C}: \boldsymbol{\varepsilon}_{(\boldsymbol{u})} d \Omega+\int_{\Gamma_{\boldsymbol{c}}} \mathbb{1}_{\mathcal{K}_{\mu}^{\circ}\left(\boldsymbol{g}_{(\boldsymbol{u})}\right)} d \Gamma \\
& -\int_{\Omega} \boldsymbol{b} \cdot \boldsymbol{u} d \Omega-\int_{\Gamma_{\boldsymbol{t}}} \boldsymbol{t}_{d} \cdot \boldsymbol{u} d \Gamma \\
\text { s.t. } & \boldsymbol{u} \in K A_{h}
\end{array}
$$

and a static approach in which a statically admissible field is obtained by minimizing the total complementary energy $(\mathrm{TCPE}) \Pi_{c}$ :

$$
\begin{array}{lc}
\min _{\boldsymbol{\sigma}} \quad \Pi_{c(\boldsymbol{\sigma})}=\int_{\Omega} \frac{1}{2} \boldsymbol{\sigma}: \mathbb{C}^{-1}: \boldsymbol{\sigma} d \Omega+\int_{\Gamma_{\boldsymbol{c}}} \mathbb{1}_{\mathcal{K}_{\mu}(\boldsymbol{\sigma} . \boldsymbol{n})} d \Gamma \\
& -\int_{\Gamma_{u}}(\boldsymbol{\sigma} \cdot \boldsymbol{n}) \cdot \boldsymbol{u}_{d} d \Gamma-\int_{\Gamma_{\boldsymbol{c}}}(\boldsymbol{\sigma} \cdot \boldsymbol{n}) \cdot\left(g_{0} \boldsymbol{n}\right) d \Gamma \\
\text { s.t. } \quad \boldsymbol{\sigma} \in S A_{h} &
\end{array}
$$

Since the error becomes zero for the optimal solution, both energies are then opposite: $\Pi_{p\left(\boldsymbol{u}^{*}\right)}=-\Pi_{c\left(\boldsymbol{\sigma}^{*}\right)}$. As a result both minimisation problems (3.3) and (3.3) provide respectively an upper bound and a lower bound of the real potential energy of the system (after inverting the sign of the complementary energy):

$$
-\Pi_{c\left(\boldsymbol{\sigma}_{h}\right)} \leq-\Pi_{c\left(\boldsymbol{\sigma}^{*}\right)}=\Pi_{p\left(\boldsymbol{u}^{*}\right)} \leq \Pi_{p\left(\boldsymbol{u}_{h}\right)}
$$

These bounds provide the engineer the capacity to judge the quality of convergence regard- 
ing the mesh since one can evaluate the proximity of approximate solutions to the exact solution. The relative difference between the upper and lower bound therefore represents the above-introduced error estimator and serves as an excellent indicator of convergence which can be defined as:

$$
\Delta E=\frac{\Pi_{p\left(\boldsymbol{u}_{h}\right)}+\Pi_{c\left(\boldsymbol{\sigma}_{h}\right)}}{\Pi_{c\left(\boldsymbol{\sigma}_{h}\right)}}=\frac{e_{\left(\boldsymbol{u}_{h}, \boldsymbol{\sigma}_{h}\right)}^{2}}{\Pi_{c\left(\boldsymbol{\sigma}_{h}\right)}}
$$

where the value of the complementary energy has been arbitrarily chosen for normalization.

\section{Finite-element discretization}

\subsection{Kinematic approach}

Displacement-type elements are used in the finite element discretization of problem (3.3). For the present case, 10-node quadratic tetrahedra will be used. Problem (3.3) can then be expressed after assembling the global stiffness matrix $\boldsymbol{K}$ and nodal force vector $\boldsymbol{f}$ as a conic minimization problem using the same formalism as in appendix B;

$$
\begin{array}{lll}
(\mathrm{TPE}) \min _{\hat{\boldsymbol{u}}, \hat{\boldsymbol{g}}} & \frac{1}{2} \hat{\boldsymbol{u}}^{T} \boldsymbol{K} \hat{\boldsymbol{u}}-\boldsymbol{f}^{T} \hat{\boldsymbol{u}} & \\
\text { s.t. } & \hat{\boldsymbol{u}}=\hat{\boldsymbol{u}}_{d} & \text { on } \Gamma_{\boldsymbol{u}} \\
& \hat{\boldsymbol{g}}=\hat{\boldsymbol{g}}_{\mathbf{0}}+\llbracket \hat{\boldsymbol{u}} \rrbracket & \text { on } \Gamma_{\boldsymbol{c}} \\
& \hat{\boldsymbol{g}} \in \mathcal{K}_{\mu}^{\circ} & \text { on } \Gamma_{\boldsymbol{c}}
\end{array}
$$

in which the primal optimization variables are $\boldsymbol{x}=\hat{\boldsymbol{u}}$ and $\boldsymbol{x}_{I}=\hat{\boldsymbol{g}}$, with $\hat{\boldsymbol{u}}$ a vector collecting all nodal degrees of freedom, and $\hat{\boldsymbol{g}}$ a vector of gaps at each node of the finite element mesh faces belonging to a contact surface $\Gamma_{c}$. Contact constraints will hence be enforced at all 6 nodes of each face with the possibility of adding additional contact points within the face in order to prevent all penetration possible due to quadratic elements. The node-to-node constraints will therefore tend to mimic a complete face-to-face constraint. After introducing a proper change of variable, the contact condition $\hat{\boldsymbol{g}} \in \mathcal{K}_{\mu}^{\circ}$ can be easily transformed into a standard second-order cone constraint using a Lorentz cone i.e.:

$$
\mathcal{K}=\mathcal{L}^{n}=\left\{(x, \boldsymbol{y}) \in \mathbb{R} \times \mathbb{R}^{n-1} \mid x \geq\|\boldsymbol{y}\|_{2}\right\}=\mathcal{K}^{*}
$$

\subsection{Static approach}

\subsubsection{Regular equilibrium elements}

Finite element discretization of problem (3.3) can be done using simple equilibrium tetrahedra. The simplest element can be obtained by considering a linear interpolation of the stress tensor components $\sigma_{i j}$ over each element. 4-node linear tetrahedra with discontinuous interpolation can be used for which continuity of the stress vector over the element facets should be explicitly written (these faces will be denoted as the set $\Sigma_{\sigma}$ ) and included as linear constraints of the corresponding optimization problem. 
Let $i=1, \ldots, 4$, be the node order index. The stress state of a point is defined by six stress components, $\boldsymbol{\sigma}^{i^{T}}=\left\{\sigma_{x x}, \sigma_{y y}, \sigma_{z z}, \sigma_{x y}, \sigma_{y z}, \sigma_{x z}\right\}$. Let $N_{i}(\boldsymbol{x})$ be the linear shape function associated with each node. The element stress state is defined by the 24 stress components of the nodes as $\boldsymbol{\sigma}^{e^{T}}=\left\{\boldsymbol{\sigma}^{1^{T}}, \ldots, \boldsymbol{\sigma}^{4^{T}}\right\}$.

We can write the stresses of an arbitrary point as:

$$
\boldsymbol{\sigma}=\left[\boldsymbol{I} N_{1}, \ldots, \boldsymbol{I} N_{4}\right] \boldsymbol{\sigma}^{e}=\boldsymbol{N} \boldsymbol{\sigma}^{e}
$$

The element compliance matrix can then be calculated as follows:

$$
\boldsymbol{F}^{e}=\int_{\Omega^{e}} \boldsymbol{N}^{T} \mathbb{C}^{-1} \boldsymbol{N} d \Omega
$$

using a 4 Gauss-points quadrature rule.

Using this equilibrium element and the typical definition for the normal projection matrix operator $\boldsymbol{P}_{N}$ on a facet of normal $\boldsymbol{n}$ and the divergence matrix operator $\boldsymbol{D}$, problem (3.3) can be expressed after assembling the global matrices as a conic minimization problem using the same formalism as appendix B

$$
\begin{array}{cll}
\text { (TPCE) } \min _{\hat{\boldsymbol{\sigma}}, \boldsymbol{t}_{c}} & \frac{1}{2} \hat{\boldsymbol{\sigma}}^{T} \boldsymbol{F} \hat{\boldsymbol{\sigma}}-\left(\boldsymbol{P}_{N} \hat{\boldsymbol{\sigma}}\right)^{T} \boldsymbol{u}_{d}-\boldsymbol{t}_{c}^{T} \hat{\boldsymbol{g}}_{\mathbf{0}} \\
\text { s.t. } & \boldsymbol{D} \hat{\boldsymbol{\sigma}}+\boldsymbol{b}=0 & \text { in } \Omega \\
& \llbracket \boldsymbol{P}_{N} \hat{\boldsymbol{\sigma}} \rrbracket=0 & \text { on } \Sigma_{\boldsymbol{\sigma}} \\
& \boldsymbol{P}_{N} \hat{\boldsymbol{\sigma}}=\boldsymbol{t}_{d} & \text { on } \Gamma_{\boldsymbol{t}} \\
& \boldsymbol{P}_{N} \hat{\boldsymbol{\sigma}}=\boldsymbol{t}_{c} & \text { on } \Gamma_{\boldsymbol{c}} \\
& \boldsymbol{t}_{c} \in \mathcal{K}_{\mu} & \text { on } \Gamma_{\boldsymbol{c}}
\end{array}
$$

in which the primal optimization variables are $\boldsymbol{x}=\hat{\boldsymbol{\sigma}}$ and $\boldsymbol{x}_{I}=\boldsymbol{t}_{c}$, with $\hat{\boldsymbol{\sigma}}$ a vector collecting all nodal stresses, and $\boldsymbol{t}_{c}$ a vector of contact surface tractions at each node of the finite element mesh faces belonging to a contact surface $\Gamma_{c}$. Contact constraints will be enforced at all 3 nodes of each face. Similarly, after introducing a proper change of variable, the contact condition $\boldsymbol{t}_{c} \in \mathcal{K}_{\mu}$ can be easily transformed into a standard second-order cone constraint using a Lorentz cone.

After implementing this method, poor performance results were obtained mainly due to the size of the problem and to linearly dependent conditions in the linear constraint matrix collecting equilibrium and traction continuity conditions. This made the problem ill-posed and caused troubles when computing the solution of the linear system equations using a direct solver. The dependencies come from the divergence condition which can be easily eliminated by integrating this condition in the definition of the finite element thus using a 21 components equivalent stress vector rather than 24 , but more importantly from the traction continuity condition over the tetrahedra faces $\Sigma_{\sigma}$. In fact, it has been shown in [47, 48, 42, 49, 40] that there exists some null eigenvalues in the compliance matrix in addition to those due to the natural rigid body modes. They correspond to zero energy 
modes called spurious kinematic modes (SKM). Illustrations of the spurious modes can be found in [48, 42]

\subsubsection{Improved equilibrium elements}

The statically admissible stress fields set $S A(\Omega)$ can be defined using an assumed stress distribution inside the elements that verifies local equilibrium with body forces [42, 48, 47. Thus the set $S A(\Omega)$ can be defined by a linear combination of a set of independent functions:

$$
\boldsymbol{\sigma}=\boldsymbol{S}+\boldsymbol{\sigma}_{0}
$$

where $\boldsymbol{S}$ are independent shape functions of degree $p$, all of them verifying equation 2.9. The columns for $\boldsymbol{S}$ can be obtained either from Morera [50] or Maxwell [51] stress potentials. The nodal variables $\boldsymbol{s}$ are called the generalized stresses. $\boldsymbol{\sigma}_{0}$ is a particular solution for the equilibrium equations, taken as equal to zero in absence of body forces. The expressions for the polynomial functions $\boldsymbol{S}$ can be found in [41].

In order to verify the other strong equilibrium form for the surface traction given by equation (2.10), the surface tractions must also be polynomial fields of degree $p$ as follows:

$$
\boldsymbol{t}=\boldsymbol{W p}
$$

where $\boldsymbol{p}$ is a vector of generalized surface tractions and $\boldsymbol{W}$ a matrix containing the shape functions of degree $p$. These discretization parameters are called connectors, since they ensure reciprocity of the surface tractions between the faces of the tetrahedra. It is possible to rewrite them in terms of the generalized stresses:

$$
p=\tilde{C} s
$$

where $\tilde{\boldsymbol{C}}$ is called the static connection matrix.

Generalized displacements $\boldsymbol{q}$, which are the conjugate of the generalized surface tractions $\boldsymbol{p}$, can be obtained as follows:

$$
\int_{\Gamma_{\boldsymbol{u}}} \boldsymbol{t}^{T} \boldsymbol{u}_{d} d \Gamma=\boldsymbol{p}^{T} \int_{\Gamma_{\boldsymbol{u}}} \boldsymbol{W}^{T} \boldsymbol{u}_{d} d \Gamma=\boldsymbol{p}^{T} \boldsymbol{q}
$$

Using these new elements, problem 4.5 can be rewritten as:

$$
\begin{array}{lll}
(\mathrm{TPCE}) & \min _{\hat{\boldsymbol{s}}, \hat{\boldsymbol{q}}} & \frac{1}{2} \hat{\boldsymbol{s}}^{T} \tilde{\boldsymbol{F}} \hat{\boldsymbol{s}}-\hat{\boldsymbol{s}}^{T} \tilde{\boldsymbol{C}}^{T} \hat{\boldsymbol{q}}-\hat{\boldsymbol{s}}^{T} \tilde{\boldsymbol{C}}^{T} \hat{\boldsymbol{q}}_{0} \\
\text { s.t. } & \boldsymbol{W} \tilde{\boldsymbol{C}} \hat{\boldsymbol{s}}=\boldsymbol{t}_{d} & \text { on } \Gamma_{\boldsymbol{t}} \\
& \tilde{\boldsymbol{C}} \hat{\boldsymbol{s}} \in \mathcal{K}_{\mu} & \text { on } \Gamma_{\boldsymbol{c}}
\end{array}
$$

with $\hat{\boldsymbol{s}}$ a vector collecting all generalized nodal stresses, $\hat{\boldsymbol{q}}$ a vector of generalized displacements and $\hat{\boldsymbol{q}}_{0}$ a vector of the equivalent generalized initial gap at each node of the finite element mesh faces belonging to a contact surface $\Gamma_{c}$. In the following, we will adopt the linear equilibrium element $(p=1)$. Contact constraints can therefore be enforced only 
at the 3 nodes of each face, ensuring by convexity that contact constraints will be verified on the whole face. Spurious kinematic modes of zero-energy are still present in this element but have been eliminated by adopting the super-element method in which each super-element is split into 4 sub-elements. Further details can be found in [42, 48].

A displacement field map can also be obtained from the generalized displacements $\hat{\boldsymbol{q}}$. Since they consist of the 3 translations at the nodes of each face (i.e. $3 \times 3$ degrees of freedom for each tetrahedron face therefore 36 in total for an element), the displacement at the super-elements tetrahedra nodes can be obtained by averaging of the displacements of the 3 converging faces at each node. However, one should note that this field does not locally verify the kinematic compatibility conditions.

\section{Interior-point method solution procedure and mesh adaptivity}

\subsection{General aspects for the primal-dual interior point method}

Since both static and kinematic approaches result in a similar mathematical formulation of second-order cone programs (see appendix B for more details), the general aspects of the interior-point method (IPM) will be described for the following second-order cone program:

$$
\begin{array}{cl}
\min _{\boldsymbol{x}, \boldsymbol{x}_{I}} & \frac{1}{2} \boldsymbol{x}^{T} \boldsymbol{P} \boldsymbol{x}+\boldsymbol{c}^{T} \boldsymbol{x}+\boldsymbol{c}_{I}^{T} \boldsymbol{x}_{I} \\
\text { s.t. } & \boldsymbol{A} \boldsymbol{x}=\boldsymbol{b} \\
& \boldsymbol{A}_{I} \boldsymbol{x}-\boldsymbol{x}_{I}=\boldsymbol{b}_{I} \\
& \boldsymbol{x}_{I} \in \mathcal{K}
\end{array}
$$

where $\boldsymbol{P}$ is a symmetrical semi-definite positive matrix and where the following rank assumptions are made:

$$
\operatorname{rank}(\boldsymbol{A})=p \quad \text { and } \quad \operatorname{rank}\left(\left[\begin{array}{ll}
\boldsymbol{P} & \boldsymbol{A}^{T}
\end{array}\right]\right)=n
$$

with $p$ being the row dimension of $\boldsymbol{A}$ and $n$ is the dimension of $\boldsymbol{x}$.

The interior point method has been shown to be well suited for solving specific types of convex programming such as linear programs (LP), second-order cone programs (SOCP) and semi-definite programming (SDP) [20, 21, 22, 23].

The idea of an IPM is to find a solution to the Karush-Kuhn-Tucker (KKT) conditions for problem (5.1) given by (B.7) by following the neighbourhood of a curve called the central path given by $\left(\boldsymbol{x}(\eta), \boldsymbol{x}_{I}(\eta), \boldsymbol{\lambda}(\eta), \boldsymbol{\nu}_{I}(\eta), \boldsymbol{s}_{I}(\eta)\right)$ and parametrized by a barrier parameter $\eta \geq 0$. This central path is no other than the unique solution to the following perturbation of the KKT system [52, 21, 22]. 
Find $\left(\boldsymbol{x}, \boldsymbol{x}_{I}, \boldsymbol{\lambda}, \boldsymbol{\nu}_{I}, \boldsymbol{s}_{I}\right)$ such that:

$$
\begin{aligned}
& \boldsymbol{P} \boldsymbol{x}+\boldsymbol{c}+\boldsymbol{A}^{T} \boldsymbol{\lambda}+\boldsymbol{A}_{I}^{T} \boldsymbol{\nu}_{I}=0 \\
& \boldsymbol{c}_{I}-\boldsymbol{\nu}_{I}-\boldsymbol{s}_{I}=0 \\
& \boldsymbol{A} \boldsymbol{x}-\boldsymbol{b}=0 \\
& \boldsymbol{A}_{I} \boldsymbol{x}-\boldsymbol{x}_{I}-\boldsymbol{b}_{I}=0 \\
& \boldsymbol{x}_{I} \circ \boldsymbol{s}_{I}=\eta \boldsymbol{e}_{I} \\
& \boldsymbol{x}_{I} \in \mathcal{K}, \boldsymbol{s}_{I} \in \mathcal{K}^{*}
\end{aligned}
$$

where in the case of Lorentz cones, $\boldsymbol{e}=(1, \mathbf{0})$ for each cone and the complementarity slackness condition $\boldsymbol{x}^{T} \boldsymbol{s}=0$ is equivalent to:

$$
\boldsymbol{x} \circ \boldsymbol{s}=\left\{\begin{array}{c}
x^{0} s^{0}+\overline{\boldsymbol{x}}^{T} \overline{\boldsymbol{s}} \\
x^{0} \overline{\boldsymbol{s}}+s^{0} \overline{\boldsymbol{x}}
\end{array}\right\}
$$

with $\boldsymbol{x}=\left(x^{0}, \overline{\boldsymbol{x}}\right)$, the $\bar{\star}$ notation representing the tail of a vector $\star$ i.e. the vector obtained by removing the first element here noted $x^{0}$. For $\eta=0$, we obtain exactly the normal KKT conditions (B.7).

The main property of the central path is that it defines a continuous set of strictly feasible points which are well-centred, i.e. far from the boundary of the feasible region except when reaching the optimum for $\eta \rightarrow 0$ (see figure 4). This will allow for large descent steps to be taken when minimizing the objective function from points the neighbourhood of the central path.

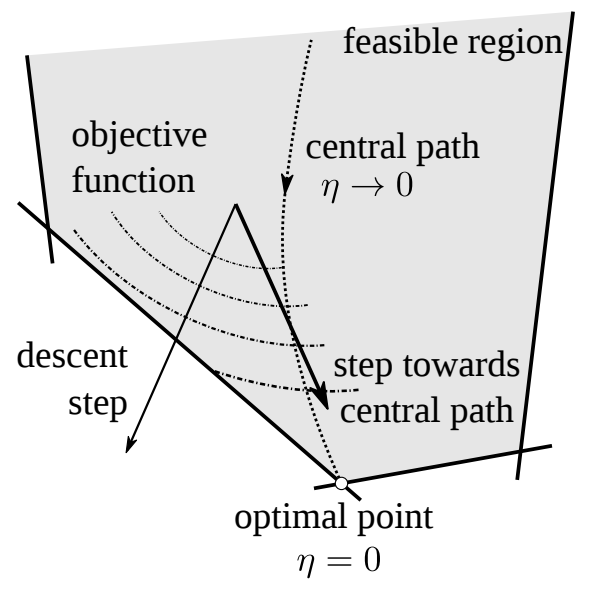

Figure 4: General idea of a IP algorithm (adapted from [29])

Most industrial IP software like Mosek [53] of SeDuMi [54], require that the quadratic form in the objective function be formulated as SOCP constraints, which can be obtained by introducing additional optimization variables [55, 53]. In order to limit the computational effort needed and taking advantage of the specific structure of the problem, we decided to keep the quadratic form thus changing some aspects of the Newton-like system to be 
solved.

The only non-linearity found in the equation set (5.3) is the complementarity condition given by (5.3e) which depends on the type of the conic constraint [52, 21, 44]. From the perturbed KKT conditions (5.3), different strategies can be chosen to compute the solutions. A typical primal interior point method is based on the elimination of the dual variables $\boldsymbol{\lambda}, \boldsymbol{\nu}_{I}$ and $\boldsymbol{s}_{I}$ and updating only the primal ones i.e. $\boldsymbol{x}$ and $\boldsymbol{x}_{I}$. We will instead use a primal-dual method which in application shows better convergence results and performance. For such an approach, dual variables remain unknown and a Newton-like step will be calculated to update all of the variables. In contrast with a feasible IPM in which all KKT conditions stated in (5.3) except (5.3e) are satisfied for each iterate, the iterates will only be required to be feasible with respect to the conic constraint $(5.3 \mathrm{f})$. In such infeasible IPM, residuals of equations (5.3a)-(5.3d) may be initially non-zero and will be driven to zero when reaching the optimal solution.

More details on the different steps of the IPM method implemented in this work can be found in [29] and are briefly summarized in appendix C.

\subsection{Mesh adaptivity}

In order to limit the number of elements used in a 3D analysis, a general adaptive remeshing scheme is implemented using the error estimator defined in equation (A.4). First, the volume contribution of the error term is computed for each element of the mesh using the dual approximate solutions $\left(\boldsymbol{u}_{h}, \boldsymbol{\sigma}_{h}\right) \in K A_{h} \times S A_{h}$ obtained from the resolution of problems (4.1) and (4.10).

Remeshing algorithms usually require a node-based metric, for that purpose the previously computed element-wise error contribution is converted to a nodal field based on error density by performing an average on all elements sharing a common node. A scalar error ratio field is therefore obtained over all nodes of the model. Finally, since the error estimator also contains surface terms over the contact area, the elementary surface term of a given contact facet is split evenly between all nodes of the contact facet and added to the previous contribution. A scalar nodal error estimator is therefore obtained.

Using an $h$-version remeshing scheme [56, 38], one can use this nodal scalar field to change the mesh size accordingly, thus improving the quality of the results while limiting the number of elements compared to a global mesh-size reduction. For this purpose, we used a general anisotropic remeshing computed on the basis of an anisotropic $3 \times 3$ metric tensor. More details about determining such an anisotropic mesh size map can be found for instance in [57]. Distene's MeshGems 3 surface (MeshGems-CADSurf) and volume (MeshGems-Tetra) are used to produce the required meshes for the finite elements analyses.

The complete procedure therefore involves the following steps:

1. an initial static and kinematic calculation are performed over an automatically softwaregenerated coarse mesh $\Omega_{h}^{(0)}$;

\footnotetext{
$\sqrt[3]{\text { http://www.meshgems.com/ }}$
} 
2. on this mesh $\Omega_{h}^{(0)}$, an error calculation is made, and global, elementary and relative errors are computed along with the relative difference between objectives functions as given by equation (3.4);

3. nodal relative errors are then used to compute a new anisotropic mesh size map;

4. a new mesh $\Omega_{h}^{(1)}$ is generated and new static and kinematic analyses are performed.

The process is repeated until a target objective difference is reached. However, this does not prevent bad quality meshes wich depends on the meshing software. A post-treatment is applied on the newly-obtained mesh in which quality measures are checked and mesh is cleaned.

Remark. Since the IPM does not really rely on good initial guesses for the optimisation variables, it is unnecessary to map the previous solution on the newly refined mesh.

\section{Illustrative applications}

\subsection{A simple example for remesh scheme with contact error}

This first example is a simple test for the error and remesh scheme using a square beam with contact conditions. The dimensions of the beam are chosen to be in agreement with the Euler-Bernoulli aspect ratio hypothesis: the main beam has a section of $0.02 \times 0.02$ $\mathrm{m}^{2}$ and a length of $0.8 \mathrm{~m}$ and underneath is a a similar section beam but with a length of $0.4 \mathrm{~m}$. The main beam is fully fixed on one end while as the second one is fully fixed at its base and right end. Unilateral contact conditions are imposed between the two beams. A downwards $2 \mathrm{kN}$ force is applied on the edge of the main beam, thus simulating a fixed beam with a pinned support at its middle. The steel material for the beam is modelled as linear elastic with Young modulus of $210 \mathrm{GPa}$ and a Poisson's ratio of 0.3. Figure 5 gives a quick description of the model. This 3D problem can be well approximated by a simple 1D Euler-Bernoulli beam model with intermediate support as sketched at the bottom of figure 5. Six remesh iterations are made and the difference between the two objective
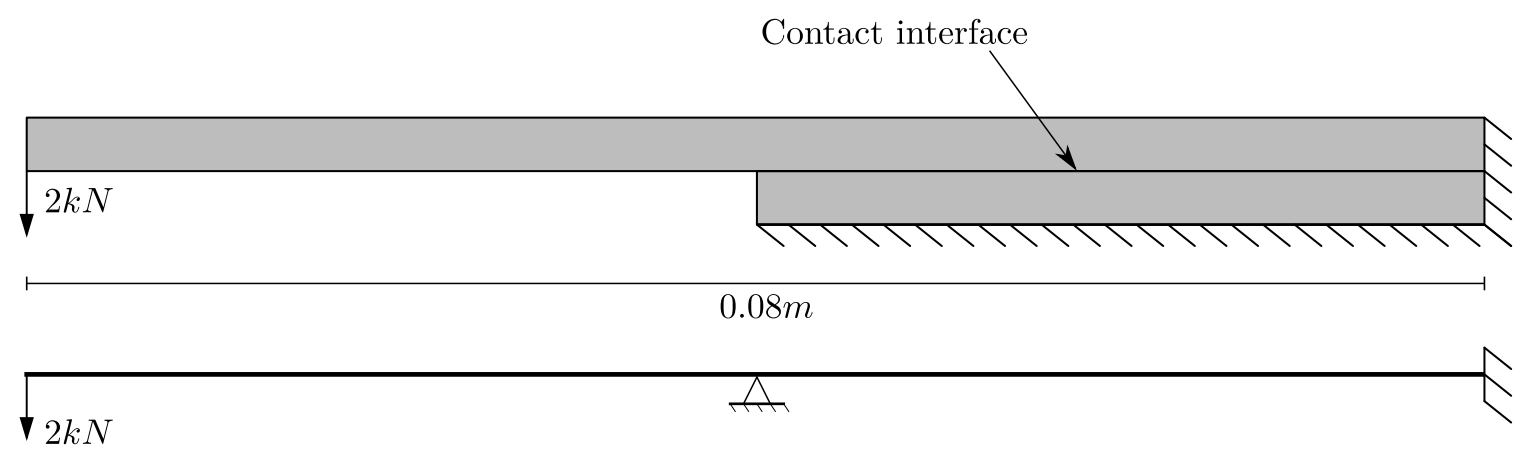

Figure 5: 3D model's description and equivalent Euler-Bernoulli beam

functions of the approaches is measured to assess the convergence quality. Figure 6 shows 


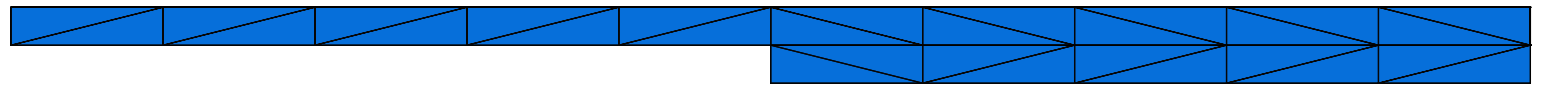

(a) Initial mesh

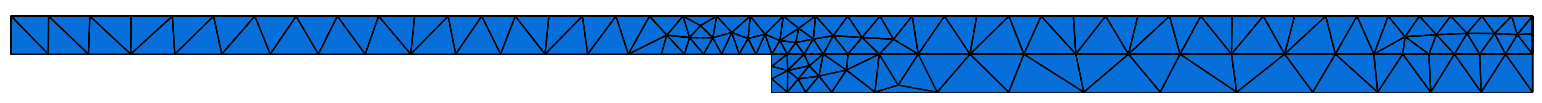

(b) Third remesh iteration

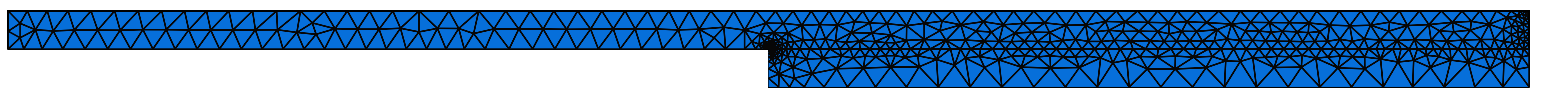

(c) Fifth remesh iteration

Figure 6: View of some of the different meshes

three of the seven different meshes used. One can clearly see the effectiveness of the remesh procedure which concentrates meshes over the contact surface between the beams. Since the relative difference computed from (3.4) gives an upper bound of the real error, the dual approach allows us to assess the global convergence quality and that by just calculating the relative difference between the two objective functions of the minimization problems.

Figure 7 a shows the evolution of this difference over the mesh iterations. We can clearly see that the after the second iteration, the difference is already less than $2 \%$ which can be considered as a good convergence level for engineering design applications. Figure $7 \mathrm{~b}$ plots the different total errors calculated over the different meshes which shows that the remesh scheme is very effective in reducing the volume error, but more importantly the surface term also which constitutes the greater contribution for this specific case.

Local quantities can also be compared such as the displacement obtained by the two approaches. Its evolution over the remeshes follows the same tendency as the errors. Indeed, the discrepancy between displacement obtained from the kinematic approach and the one post-processed from the static approach tend to close and eventually become indistinguishable at the fourth iteration as shown in figure $7 \mathrm{c}$. The maximum displacement at the tip of the beam is also in accordance with the analytical value of $2.66 \mathrm{~cm}$ obtained from the simple 1D beam model of figure 5. Plots of the deformed mesh and the normal gap are also given in figures 8 and 9 .

\subsection{Performance and results comparison with Abaqus}

The next example aims at assessing the procedure computational performance with respect to computations made using the Abaqus software [45]. The considered problem is a beam-column continuous connection frequently found in steel-structures buildings, the constitutive material being the same as before. To eliminate all forms of variations and to focus on the performance of the solvers only, the same series of 6 iteratively refined meshes is used in all the studies, both for our implementation and for Abaqus computations. This model is calculated using the IP algorithm and various configurations in Abaqus, 


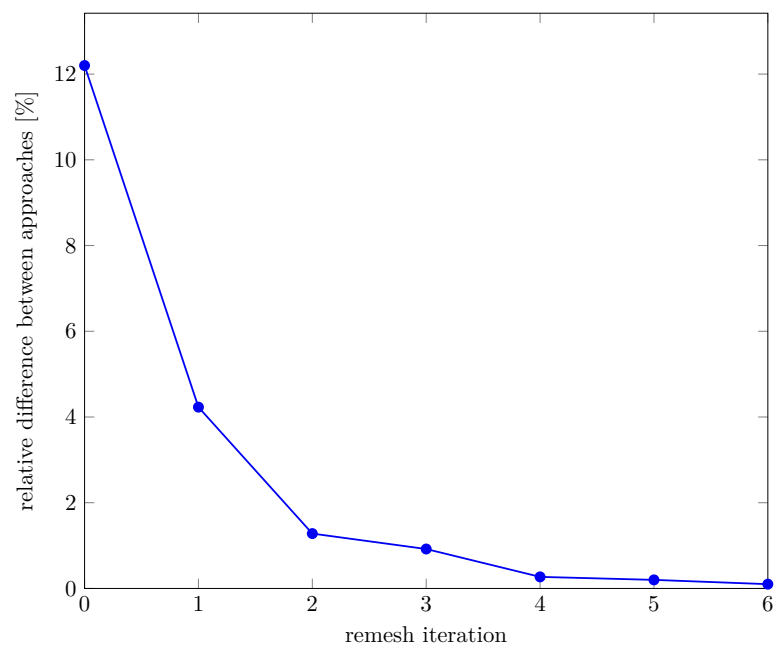

(a) Relative difference between energies

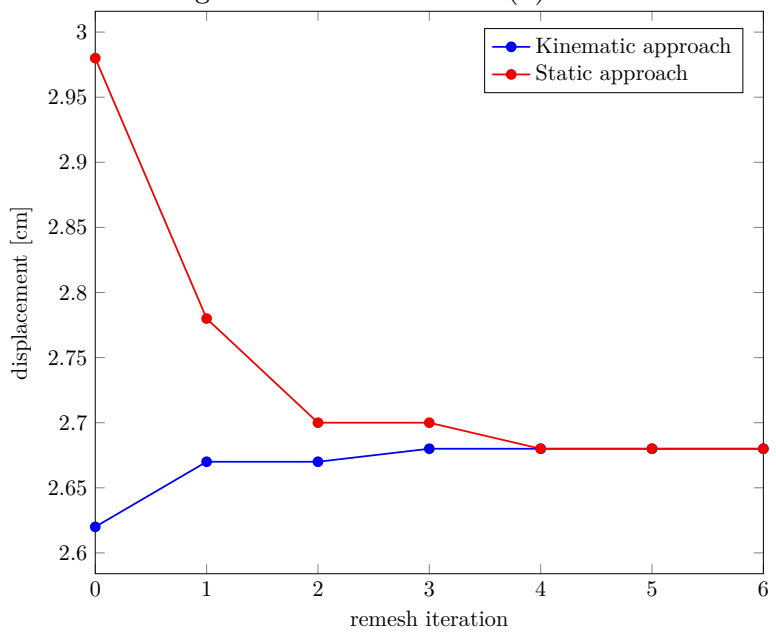

(c) Maximum vertical displacement at the tip of the first beam

Figure 7: Convergence plots for the first example

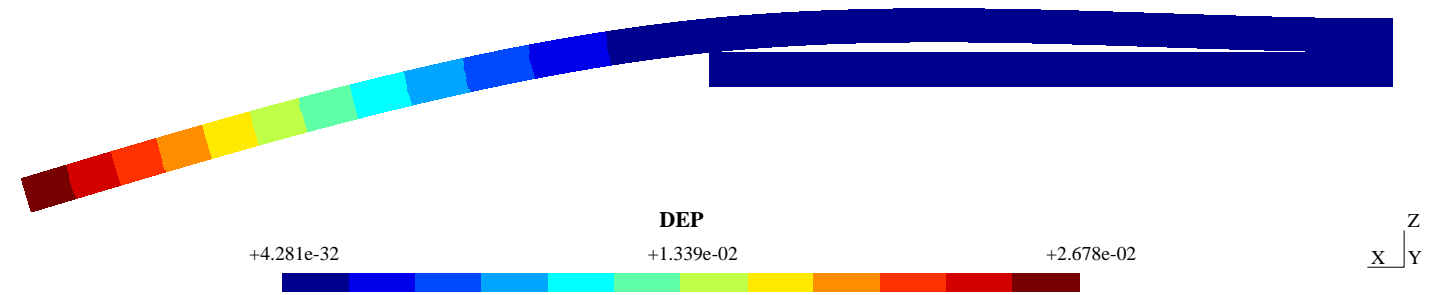

Figure 8: Deformed shape and displacement magnitude (in meters) for the fourth remesh iteration 


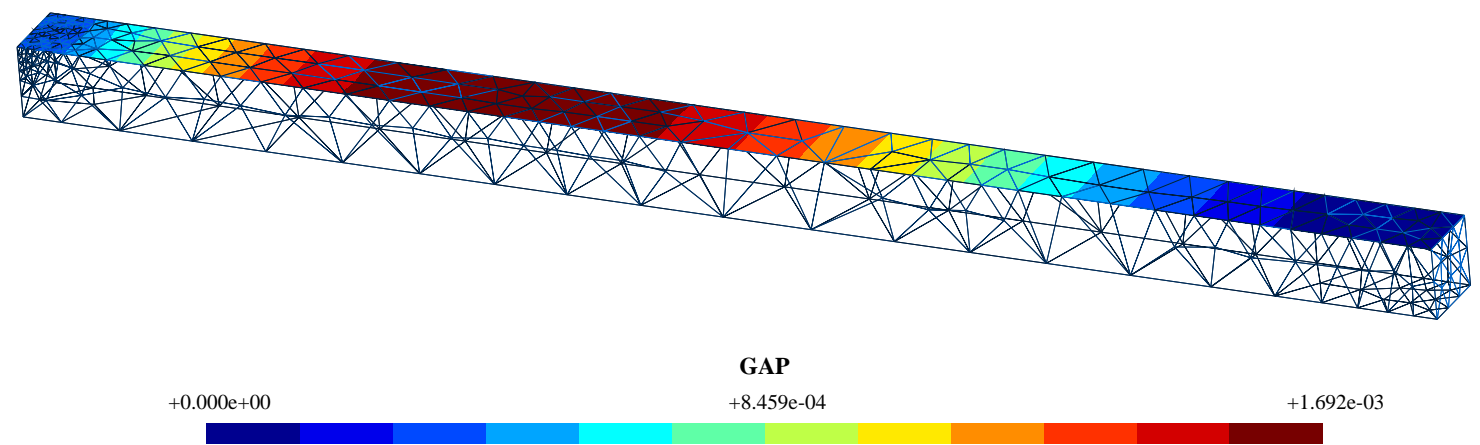

Figure 9: Normal gap (in meters) on the contact surface between the two beams

corresponding to different modelling choices of contact enforcement summarized in table 1 . Note that these choices have been made in order to position the IPM with respect to a very simple and general-purpose enforcement of contact constraints (penalty approach) as well as a more advanced one (Augmented Lagrangian). For the latter case, additional contact points other than the nodes are used by Abaqus in the surface-to-surface computation. For the comparison to be fair, we also added 4 additional points inside each triangular facets for contact enforcement in our implementation, thereby reducing the potential violation of contact conditions in the node-to-node formulation inside the facet. With such additional contact points, similar system sizes are obtained. Default parameters have been retained for assessing the robustness of our approach with respect to its use by an inexperienced user.

Two variants are defined by changing the friction coefficient between the beam's endplates and the column's flanges:

- a frictionless contact is used for model 1 (only unilateral contact conditions are enforced so that the comparison is most relevant);

- a $\mu=0.3$ friction coefficient is used for model 2 (note that Abaqus solves standard Coulomb friction)

Figure 10 gives a general description of the model while three mesh iterations are represented on figure 11. It consists of a HEB200 central column with two IPE360 beams attached over the flanges using welded end plates and bolts. The end plates have a $15 \mathrm{~mm}$ thickness and 6 M18 bolts are used to attach each beam. Web stiffeners are used to prevent its buckling. For the HEB column, their thickness is $12.7 \mathrm{~mm}$ to coincide with the IPE's flange thickness, whereas for the IPE beams, $15 \mathrm{~mm}$ thick stiffeners are used over the supports to correctly channel shear forces. The stiffeners are glued to the beams and column using tie constraints (*TIE) in Abaqus. We suppose that the bolt hole is equal to its diameter and, to prevent rigid body motions, one of the bolt heads is glued to the plate. A $45 \mathrm{MPa}$ normal pressure is applied over the top section of the HEB column and 


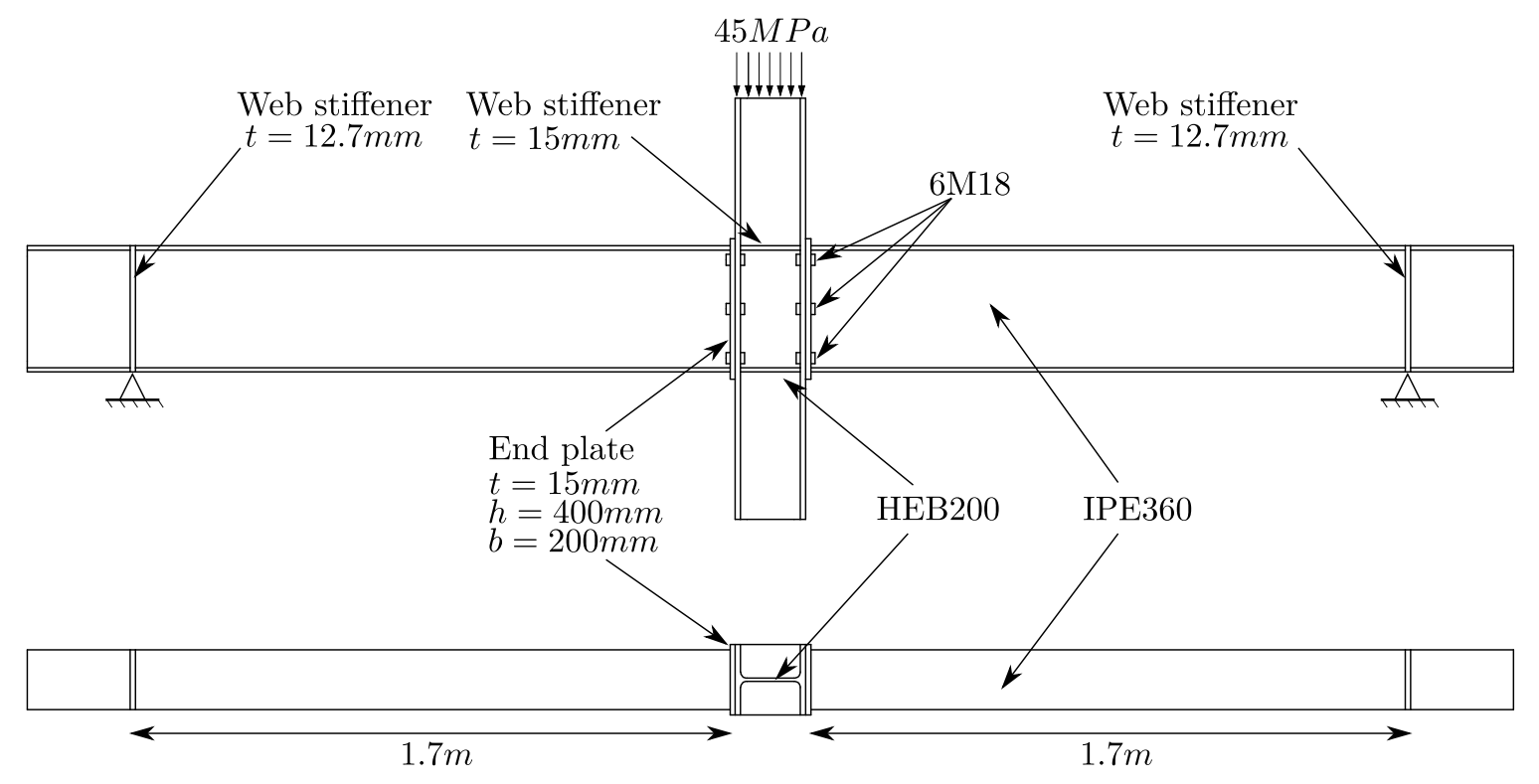

Figure 10: Description of the used model

the displacement of the nodes belonging to the surface defined by the intersection of the IPE's web stiffeners are blocked in all the 3 directions, the HEB sections remaining free.

Table 1: Definition of contact modelling choices in Abaqus

\begin{tabular}{lcccc}
\hline & Model 1-1 & Model 1-2 & Model 2-1 & Model 2-2 \\
\hline Mesh type & C3D10 & C3D10 & C3D10 & C3D10 \\
\hline Contact pairs type & node to surface & surface to surface & node to surface & surface to surface \\
Use supplementary contact points & No & Yes & No & Yes \\
\hline Normal behaviour & Hard contact & Hard contact & Hard contact & Hard contact \\
Enforcement method & Penalty & Augmented Lagrangian & Penalty & Augmented Lagrangian \\
Stiffness reduction & 0.01 & No reduction & 0.01 & No reduction \\
\hline Tangential behaviour & Frictionless & Frictionless & Frictional & Frictional \\
Enforcement method & & & Penalty & Penalty \\
Friction coefficient & & & 0.30 & 0.30 \\
\hline
\end{tabular}

Since Abaqus offers displacement-based elements only, the calculation times are compared with respect to the kinematic approach. Since the same meshes are used in Abaqus, the solved system size is nearly the same, the system sparsity patterns and conditioning are also comparable. The main difference lies in contact modelling and resolution strategy. In order to closely compare the numerical performance of the algorithm, all the calculations were made using the same machine. OpenMP technology was used to parallelize over 8 threads. Table 2 summarizes the machine's specifications.

Tables 3 and 4 show the CPU times for the models described in table 11. The IPM is largely comparable to Abaqus penalty approach (PEN) in terms of CPU times, with even 


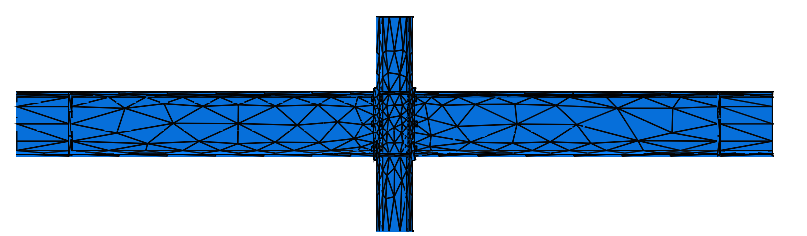

(a) Initial mesh

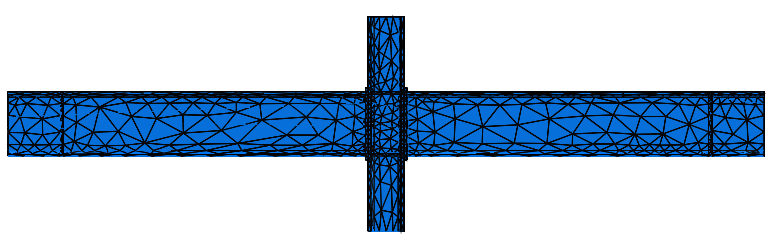

(b) First remesh iteration

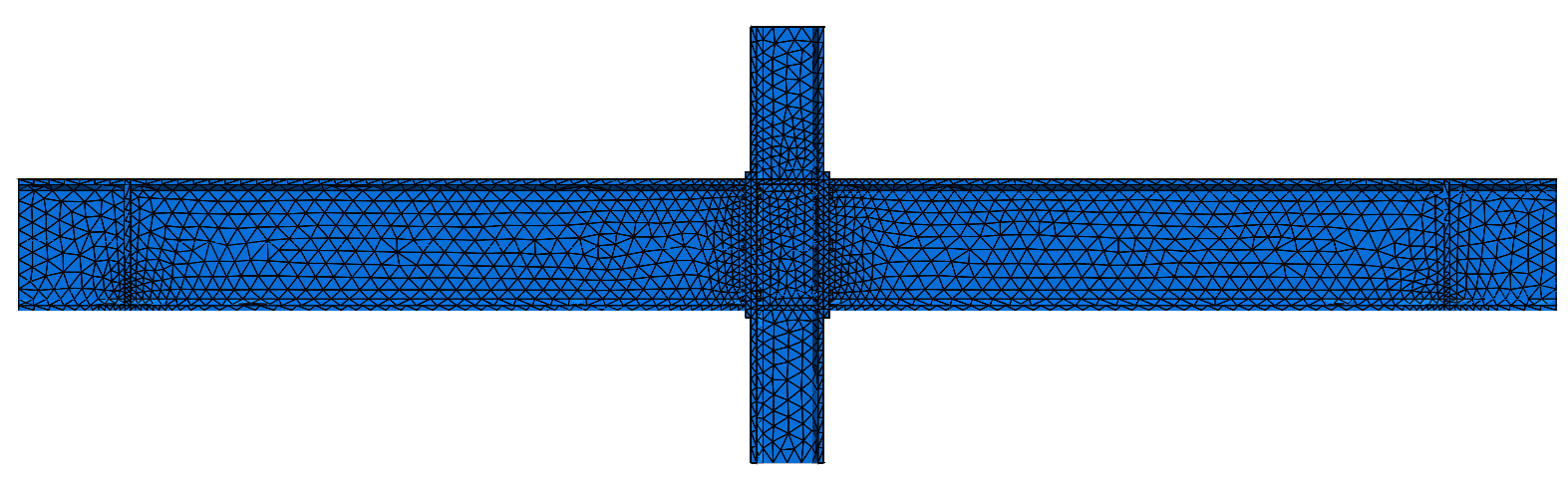

(c) Third remesh iteration

Figure 11: View of some of the different meshes

Table 2: Computer specifications

\begin{tabular}{ll}
\hline Processor & Intel core i7-4700MQ \\
Number of cores & 4 \\
Number de threads & 8 \\
Base frequency & $2.40 \mathrm{GHz}$ \\
Maximum frequency & $3.40 \mathrm{GHz}$ \\
Cache & $6 \mathrm{MB}-\mathrm{SmartCache}$ \\
RAM & $16.0 \mathrm{~GB}$ (15.7 GB usable) \\
\hline
\end{tabular}

Table 3: First model (frictionless contact) CPU times and speed-up factors of the IPM over Abaqus augmented Lagrangian approach and penalty approach

\begin{tabular}{ccccccc}
\hline $\begin{array}{c}\text { Remesh } \\
\text { iteration }\end{array}$ & $\begin{array}{c}\text { Mesh } \\
\text { Size }\end{array}$ & $\begin{array}{c}\text { IPM kinematic } \\
\text { approach } \\
(\mathrm{s})\left(N_{\text {iter }}\right)\end{array}$ & $\begin{array}{c}\text { Abaqus AL } \\
\text { approach } \\
(\mathrm{s})\left(N_{\text {iter }}\right)\end{array}$ & $\begin{array}{c}\text { Speed-up } \\
\text { factor }\end{array}$ & $\begin{array}{c}\text { Abaqus PEN } \\
\text { approach } \\
(\mathrm{s})\left(N_{\text {iter }}\right)\end{array}$ & $\begin{array}{c}\text { Speed-up } \\
\text { factor }\end{array}$ \\
\hline 0 & $N_{e}=9150$ & $10.2(17)$ & $25.0(21)$ & 2.5 & $9.0(8)$ & 0.9 \\
1 & $N_{e}=33051$ & $28.3(18)$ & $69.0(22)$ & 2.4 & $30.0(9)$ & 1.1 \\
2 & $N_{e}=42696$ & $35.0(17)$ & $97.0(24)$ & 2.8 & $38.0(9)$ & 1.1 \\
3 & $N_{e}=85187$ & $78.8(17)$ & $278.0(25)$ & 3.5 & $98.0(9)$ & 1.2 \\
4 & $N_{e}=222082$ & $320.6(18)$ & $1368.0(30)$ & 4.3 & $435.0(10)$ & 1.4 \\
\hline
\end{tabular}


Table 4: Second model (frictional contact) CPU times and speed-up factors of the IPM over Abaqus augmented Lagrangian approach and penalty approach

\begin{tabular}{ccccccc}
\hline $\begin{array}{c}\text { Remesh } \\
\text { iteration }\end{array}$ & $\begin{array}{c}\text { Mesh } \\
\text { Size }\end{array}$ & $\begin{array}{c}\text { IPM kinematic } \\
\text { approach } \\
(\mathrm{s})\left(N_{\text {iter }}\right)\end{array}$ & $\begin{array}{c}\text { Abaqus AL } \\
\text { approach } \\
(\mathrm{s})\left(N_{\text {iter }}\right)\end{array}$ & $\begin{array}{c}\text { Speed-up } \\
\text { factor }\end{array}$ & $\begin{array}{c}\text { Abaqus PEN } \\
\text { approach } \\
(\mathrm{s})\left(N_{\text {iter }}\right)\end{array}$ & $\begin{array}{c}\text { Speed-up } \\
\text { factor }\end{array}$ \\
\hline 0 & $N_{e}=9150$ & $9.8(18)$ & $37.0(23)$ & 3.8 & $14.0(8)$ & 1.4 \\
1 & $N_{e}=33051$ & $29.8(18)$ & $116.0(24)$ & 3.9 & $47.0(10)$ & 1.6 \\
2 & $N_{e}=42696$ & $40.0(18)$ & $133.0(21)$ & 3.3 & $57.0(9)$ & 1.4 \\
3 & $N_{e}=85187$ & $78.8(18)$ & $445.0(25)$ & 5.6 & $165.0(9)$ & 2.1 \\
4 & $N_{e}=222082$ & $334.1(19)$ & $2296.0(29)$ & 6.9 & $760.0(10)$ & 2.3 \\
\hline
\end{tabular}

a small speed-up factor when compared to the augmented Lagrangian (AL) approach. Compared to the latter, which generally yields more accurate results, the IPM shows a speed-up factor of 2 to 4 in frictionless contact and up to 6 in frictional contact, the speedup factor increasing with the mesh size. This is an attractive feature of the IPM since it can handle large scale sparse problems efficiently. While Abaqus number of iterations required for convergence tends to increase (from 20 to 30 for the AL approach), the IPM number of iteration remains fairly constant varying between 18 and 20 iterations for all the models and remeshes, this almost independence of number of iteration with respect to problem size is a known attractive feature of IPM methods. Figures 12 and 13 respectively illustrate the total CPU cost and the average CPU cost per inner iteration of the non-linear solvers for the different configurations.

Let us mention that the dual solution with equilibrium elements exhibits approximately the same computation time as the primal one since the linear system is roughly the same size. Since both problems are independent, they can be run in parallel. Finally, the error calculation being a simple post-processing step, it takes less than $1 \%$ of the time needed for the whole resolution.

To assess the global convergence level with mesh refinement, total quantities are considered. The influence of mesh size over the relative difference between the static and kinematic IP approaches has been represented in figures 14 and 15 . The quality of convergence of the finite element analysis can therefore be directly evaluated by checking the relative difference between the approaches which reaches acceptable values (less than $5 \%$ directly after the first remesh iteration) and $1.68 \%$ for the frictionless model and $1.2 \%$ for the frictional model on the fourth remesh iteration. When inspecting the convergence rate of the global error when refining the mesh, we observe that it is roughly linear with respect to the number of degrees of freedom.

Let us now consider some local quantities. Figure 16 shows the evolution of the total vertical displacement of the column over the six meshes. We can clearly see the upper and lower bound offered by the dual approach that we used. Abaqus AL approach shows nearly the same results as the kinematic approach whereas with the penalty contact constraints, one can easily over-estimate displacements thus leading to misinterpretations. These aspects are generally unknown to engineers who do not have means to estimate an upper 


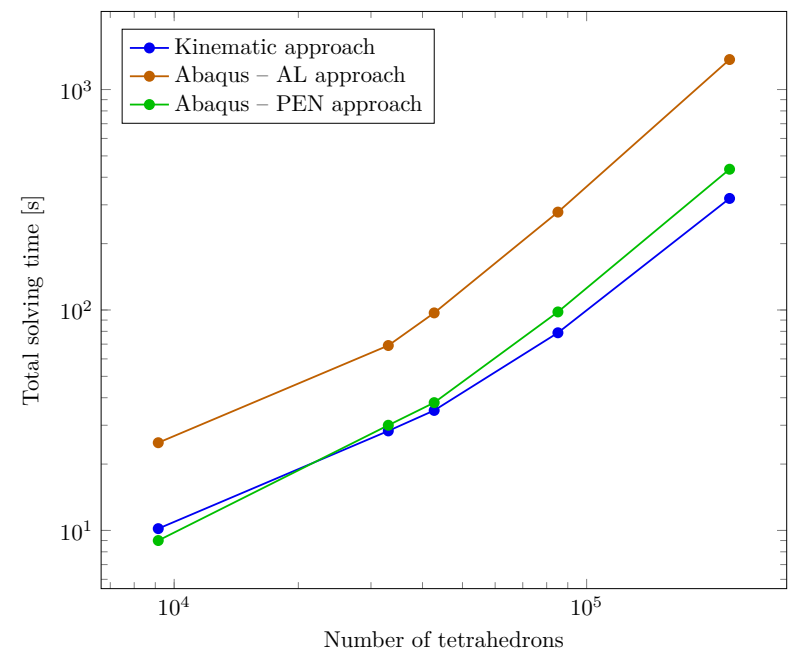

(a) Model 1 - Frictionless contact

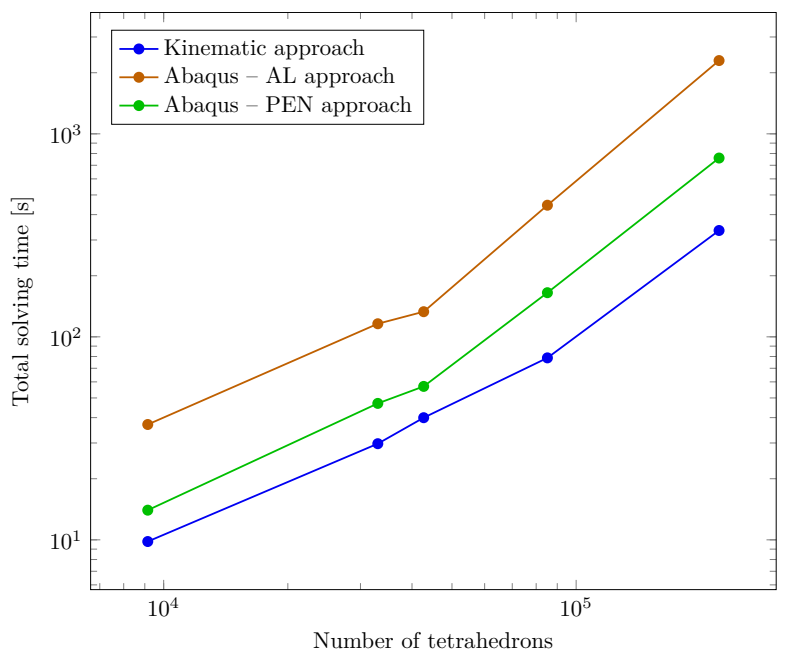

(b) Model 2 - Frictional contact

Figure 12: Total solving time for each mesh size

bound for displacements, contrary to what offers equilibrium-based computations. The maximum gap between the end plate and the column's flange presented in figure 17 shows the consequences of the contact constraints enforcement method. It is clear that with the penalty, the maximum gap is overestimated. An important point is that with the IPM, no contact constraint violation is permitted, thus we have zero penetration, whereas in Abaqus, a penetration tolerance is allowed. The results show that a "negative" gap, equivalent to up to $8 \%$ of the maximum gap, is possible with the penalty approach $(-0.055 \mathrm{~mm}$ compared to $0.707 \mathrm{~mm}$ for the first mesh in model 1 as seen in figure $18 \mathrm{c}$ and $-0.058 \mathrm{~mm}$ compared to $0.697 \mathrm{~mm}$ for the first mesh in model 2 as seen in figure 20c. However, with the AL approach, negative gaps are limited to $0.0001 \%$ of the maximum gap, thus yielding better results that are comparable to the IP kinematic approach. With the penalty approach in Abaqus and the removal of supplementary contact constraints, one can manage to obtain results in a more reasonable time. However, the quality of the results is poor and contact conditions are generally over-evaluated or violated as shown in figure 17 and in the gap iso-values shown in figures 18 to 21 (COPEN being the gap keyword in Abaqus).

It must be noted that in the case of frictionless contact, both our model and Abaqus impose the same Signorini unilateral conditions so that the comparison is relevant. However, the validity of the comparison between the models including friction can be questioned because of our choice of associated friction. In particular, it can be expected that both computations will differ, especially regarding the normal and tangential components of the gap vector since associated friction predicts normal opening during sliding. Nevertheless, due to the end-plates flexural behaviour considered in this example, surfaces in sliding are quite limited in size (they approximately correspond to the black region of Figure 21b) and the maximum tangential gap amplitude is less than $5 \%$ of the maximum normal gap. The impact of associated friction is therefore quite limited for this specific situation. Figures 22 and 23 show the local contact pressure over the end plates, one obtained using 


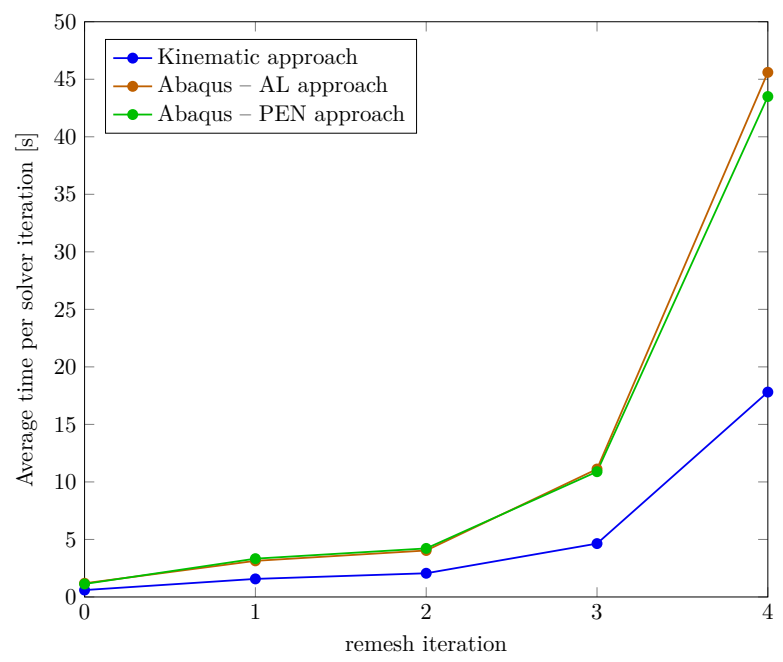

(a) Model 1 - Frictionless contact

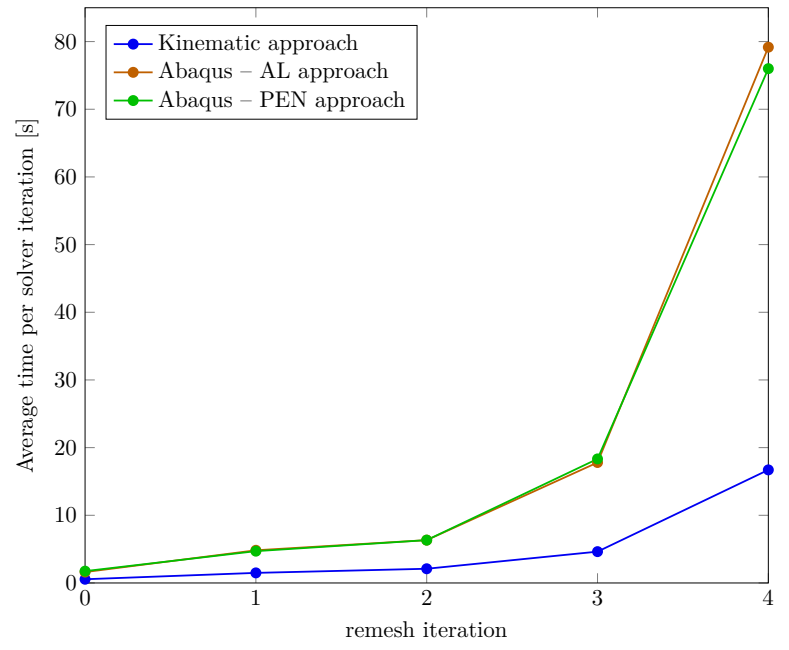

(b) Model 2 - Frictional contact

Figure 13: Average solving time per internal algorithm iteration for each mesh iteration

the IP static approach and the two other from Abaqus (CPRESS being the normal pressure keyword in Abaqus). We can clearly see that the stresses post-processed by Abaqus from a displacement field are underestimated. While the AL approach gives comparable results to the IP static approach (247 MPa compared to $268 \mathrm{MPa}$ for model 1 and $243 \mathrm{MPa}$ compared to $261 \mathrm{MPa}$ for model 2, again, despite the modelling difference of frictional behaviour), the penalty approach largely underestimates these values (89.2 $\mathrm{MPa}$ for model 1 and 87.3 MPa for model 2). Again, close agreement between our computations and Abaqus AL computations in terms of normal pressure values is found due to a limited impact of associated friction in this bending-dominated case. However, the comparison should be made with greater care in the case of compressed end plates with an important torsional loading for instance, where the tangential behaviour would be dominant. These aspects will be investigated in further studies in which non-associated frictional contact will be considered. In any case, relying on a dual approach enables to produce results of good quality in a robust and computationally efficient manner which is very attractive in the context of design engineering. 


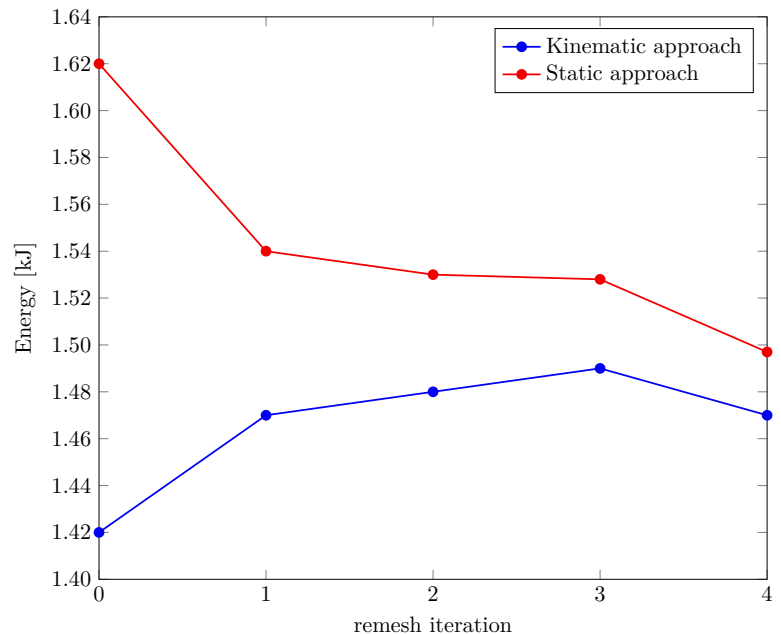

(a) Model 1 - Frictionless contact

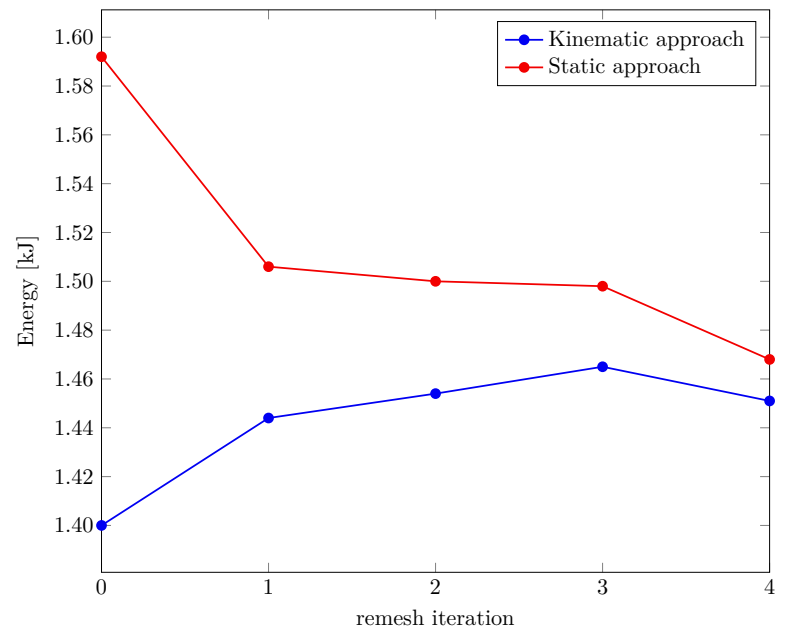

(b) Model 2 - Frictional contact

Figure 14: Objective function values for the static and the kinematic IP approach

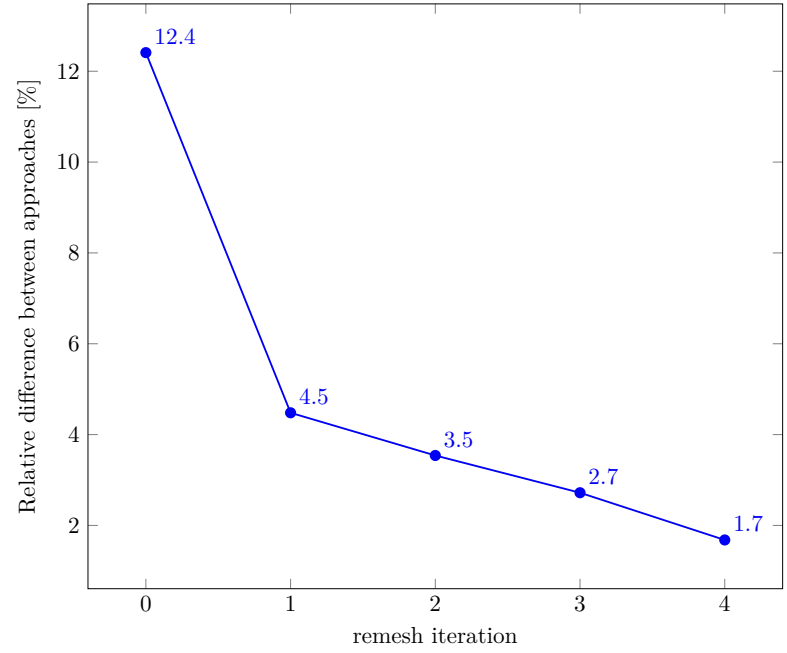

(a) Model 1 - Frictionless contact

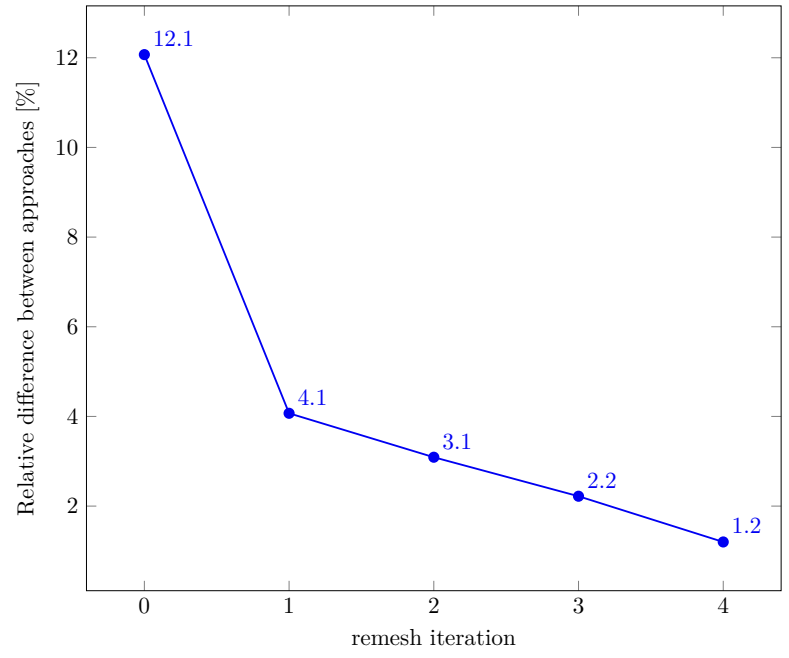

(b) Model 2 - Frictional contact

Figure 15: Relative difference between static and kinematic IP approach's objective functions 


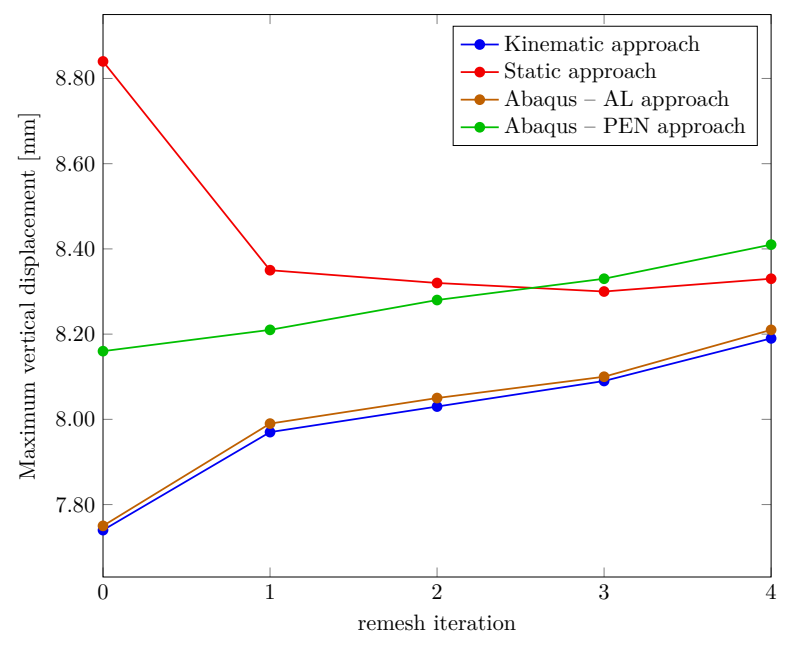

(a) Model 1 - Frictionless contact

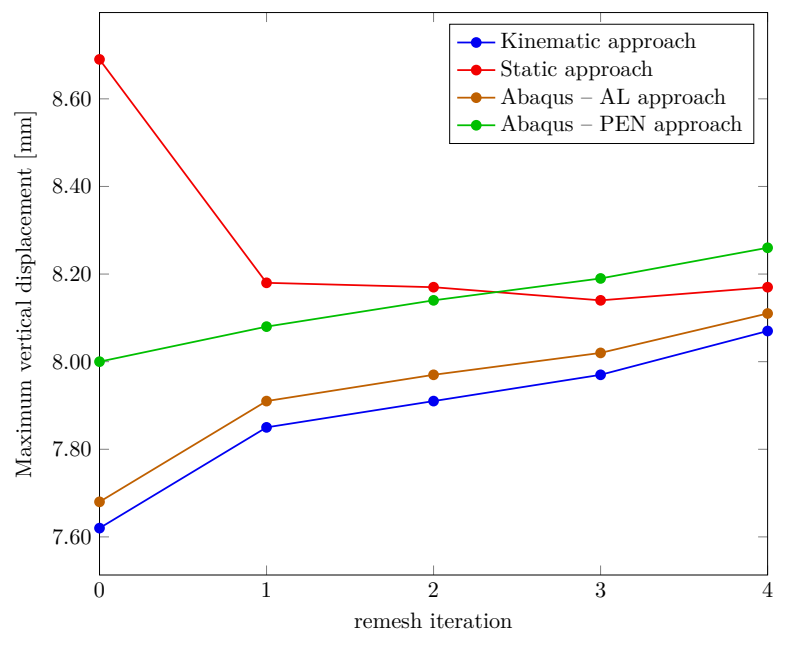

(b) Model 2 - Frictional contact

Figure 16: Total vertical displacement of the column

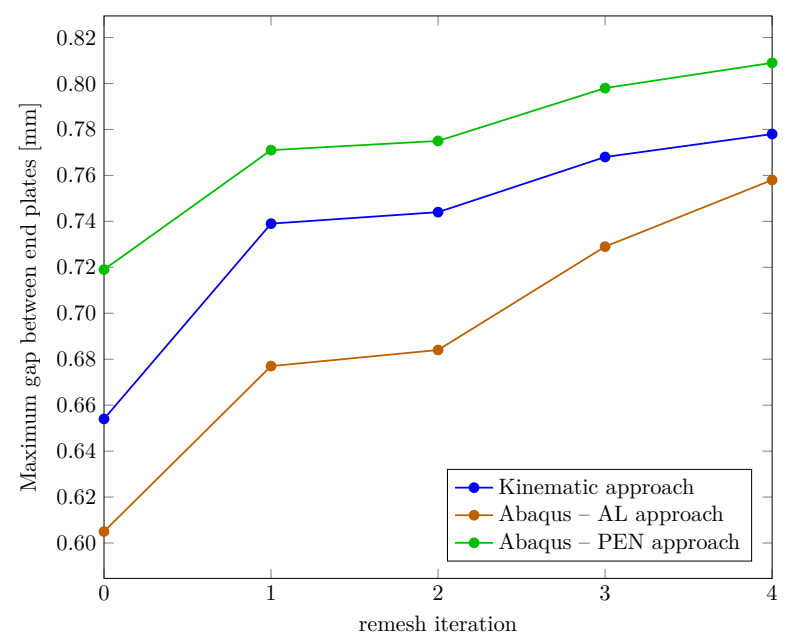

(a) Model 1 - Frictionless contact

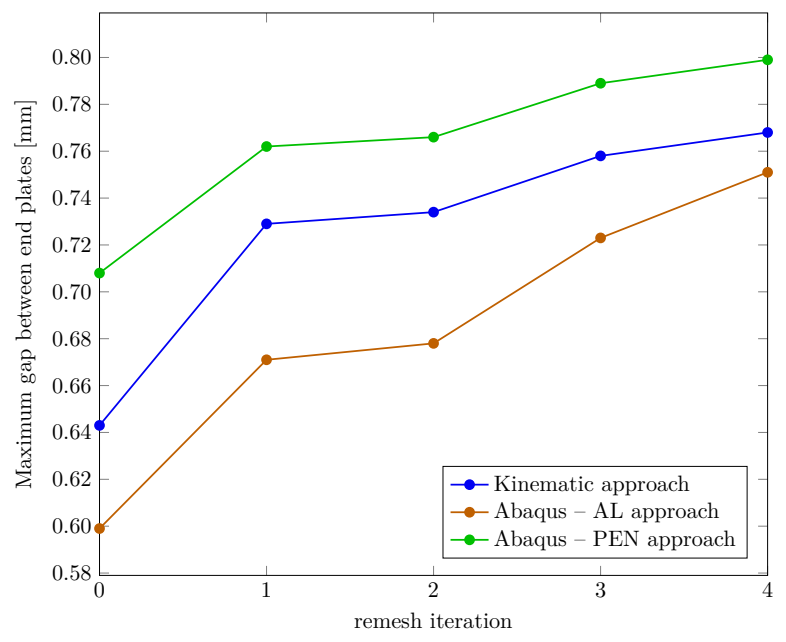

(b) Model 2 - Frictional contact

Figure 17: Maximum gap between the end plate and the column's flange 


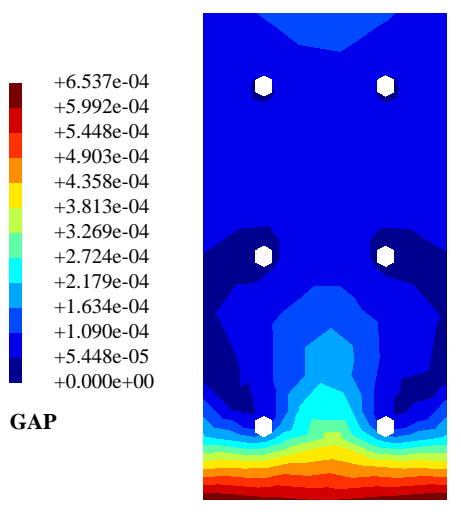

(a) IPM kinematic approach

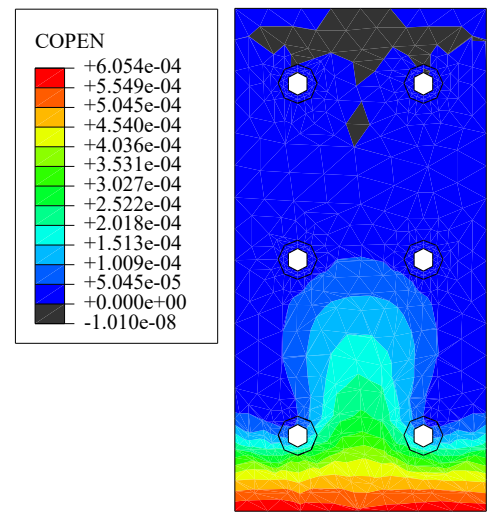

(b) Abaqus AL approach

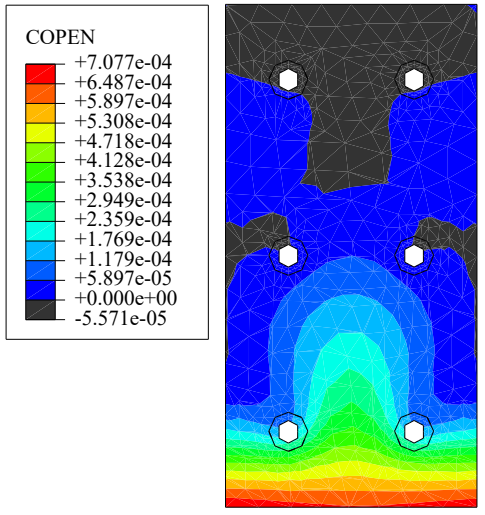

(c) Abaqus PEN approach

Figure 18: Model 1 - frictionless contact, initial coarse mesh, gap iso-values in (m) over one of the endplates

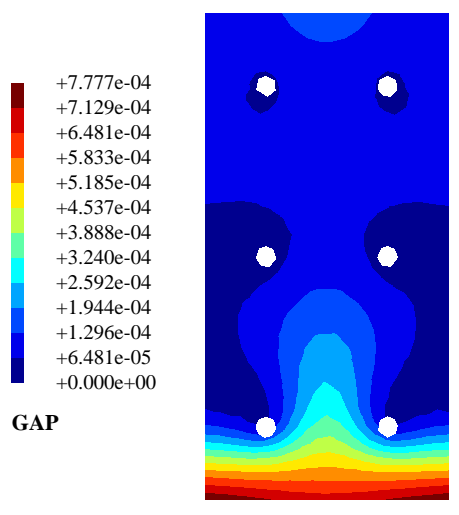

(a) IPM kinematic approach

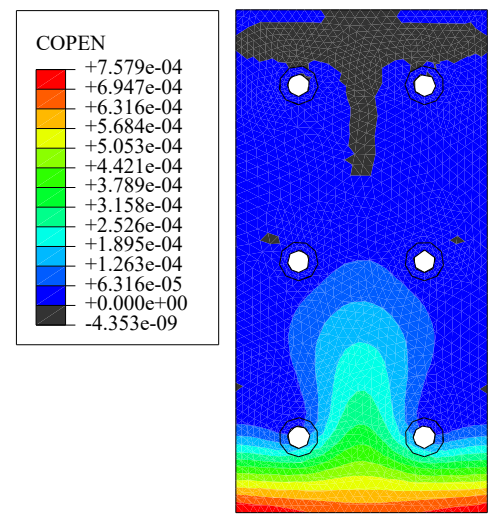

(b) Abaqus AL approach

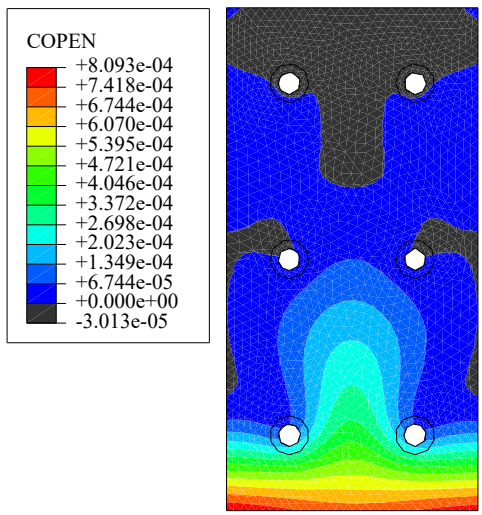

(c) Abaqus PEN approach

Figure 19: Model 1 - frictionless contact, fourth remesh iteration, gap iso-values in (m) over one of the end-plates

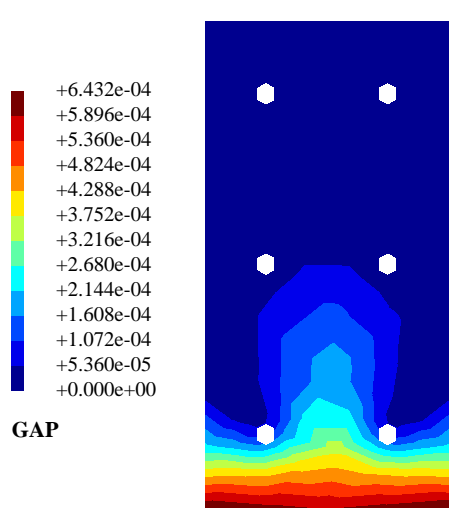

(a) IPM kinematic approach

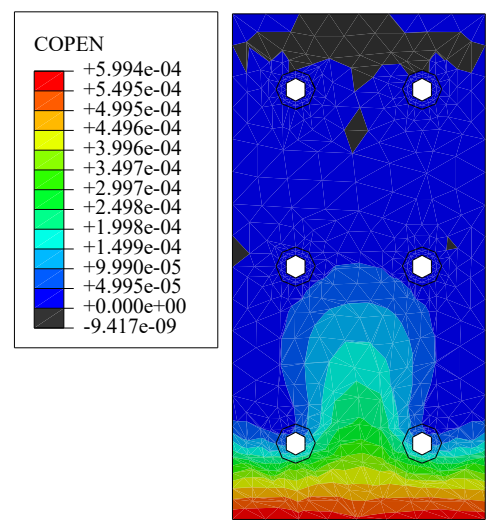

(b) Abaqus AL approach

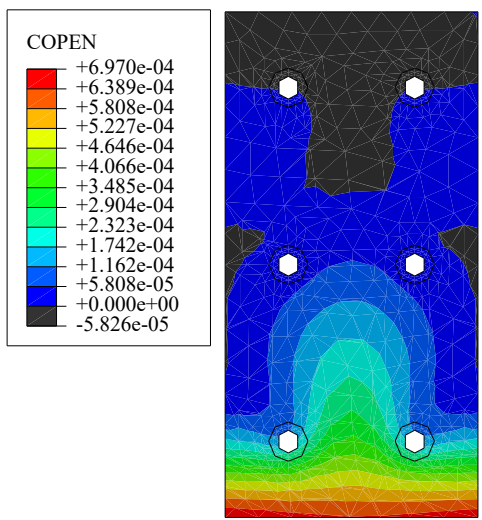

(c) Abaqus PEN approach

Figure 20: Model 2 - frictional contact, initial coarse mesh, gap iso-values in (m) over one of the end-plates 


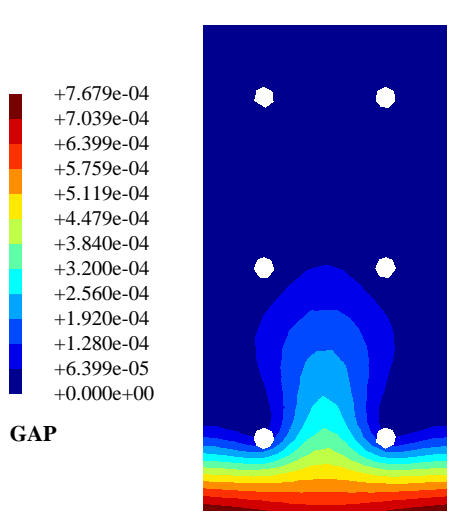

(a) IPM kinematic approach

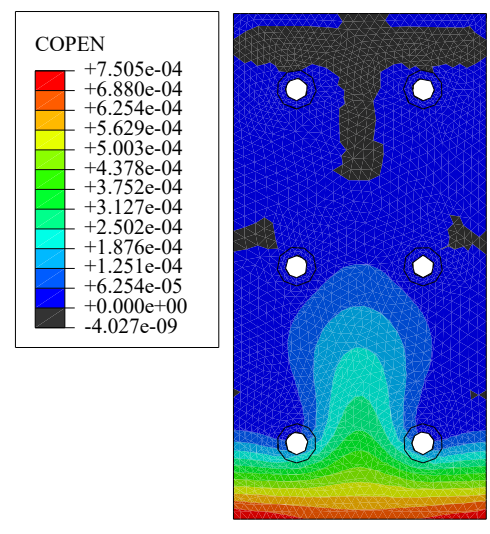

(b) Abaqus AL approach

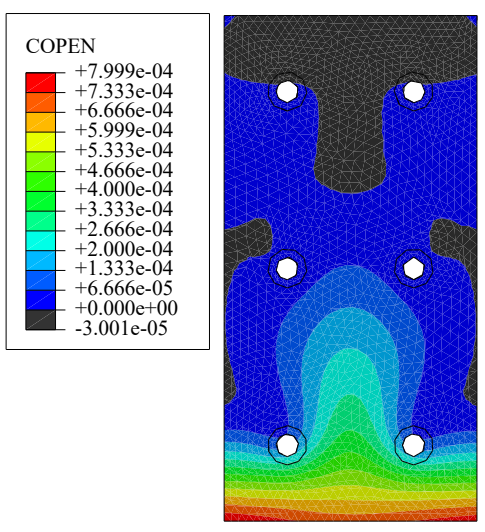

(c) Abaqus PEN approach

Figure 21: Model 2 - frictional contact, fourth remesh iteration, gap iso-values in (m) over one of the end-plates

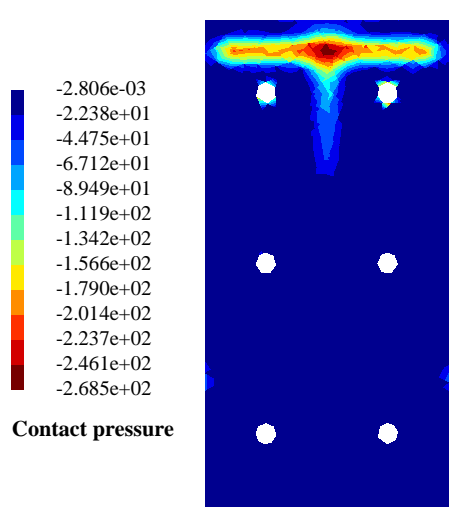

(a) IPM static approach

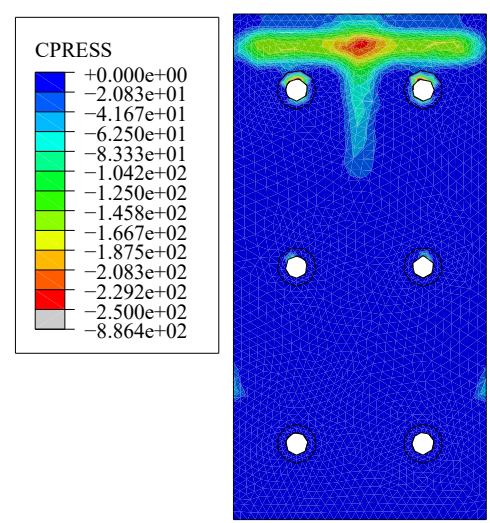

(b) Abaqus AL approach

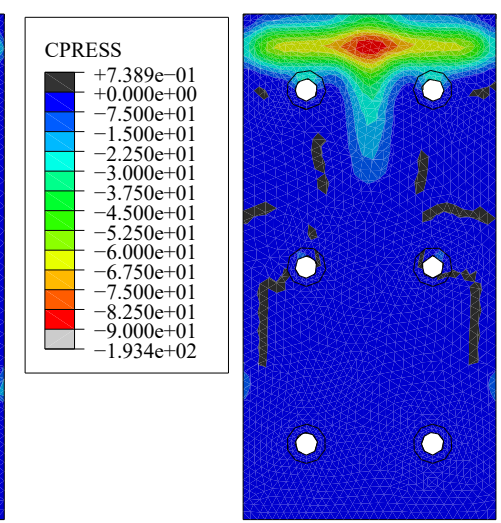

(c) Abaqus PEN approach

Figure 22: Model 1 - frictionless contact, fourth remesh iteration, normal pressure iso-values in (MPa) over one of the end-plates

\section{Conclusions and perspectives}

Modelling three-dimensional elastic structures in contact under static loads using SOCP and IPM has been investigated in this paper. Using a dual principle approach, a kinematically admissible displacement field and statically admissible stress field can be obtained by solving a pair of minimisation problems. The obtained second-order cone optimization problems are solved using a primal-dual interior point method for which convergence properties are ensured [44]. The optimal pair of variables allows for computing an error estimator based on dual analysis which is then used to produce a new anisotropic mesh size map. The whole process of solving the minimization problems, calculating errors and remeshing, has been automatized. The process is repeated until a target relative difference between objective functions is reached. Since this difference measures the global quality of convergence of the two approaches and provides a upper bound estimate of the global 


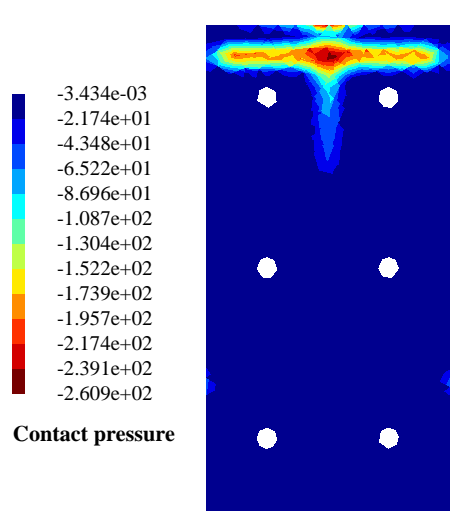

(a) IPM static approach

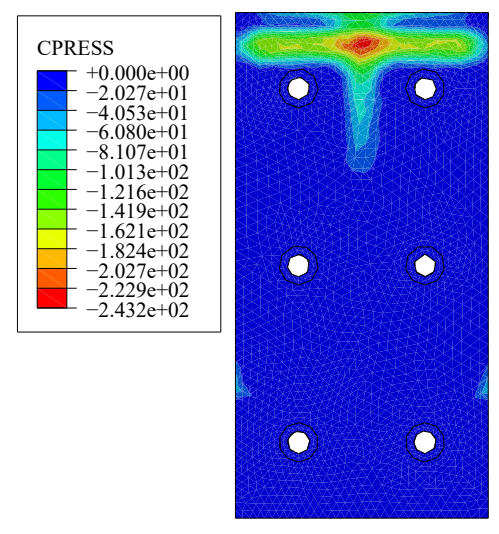

(b) Abaqus AL approach

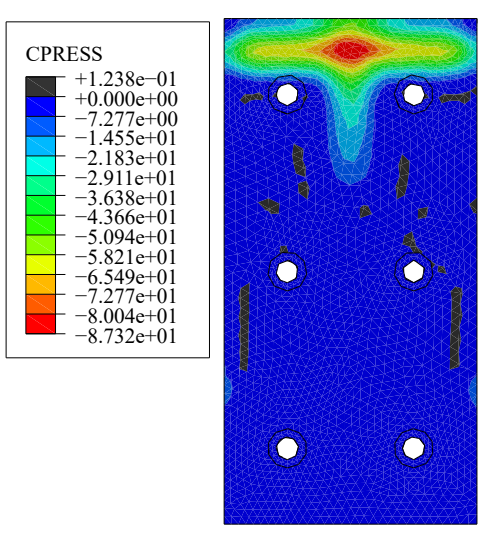

(c) Abaqus PEN approach

Figure 23: Model 2 - frictional contact, fourth remesh iteration, normal pressure iso-values in (MPa) over one of the end-plates

error, it is an excellent indicator of the convergence of the finite elements computations.

The two examples presented in this paper show the numerical and technical efficiency of this process. The error estimator which consists of an elementary volume term and a local surface contribution due to contact complementarity condition violation enables to remesh areas of interest while limiting the number of elements. Few remesh iterations are generally enough to ensure a difference under $2 \%$, this number can be reduced if the coefficient of error reduction used in the remesh algorithm is increased and if the initial mesh is less coarse. However, the mesh size map will be more difficult to process by automatic mesh generators which could produce bad quality meshes.

The IPM capability to take into account a great number of conic constraints shows all its advantages over traditional penalty or augmented Lagrangian approaches. Excellent results were obtained for large scale problems, with a relatively constant number of iterations ranging between 17 and 20 while the number of iterations in Abaqus AL approach tended to increase with the problem size. At the expense of quality, contact problems can be solved with nearly the same time as the IPM using Abaqus penalty approach. However, it is known that enforcing contact conditions with such a general-purpose strategy can yield poor estimates of stress levels and larger displacements in structures. The IPM dual approach appears therefore as an interesting alternative, offering high-quality displacement and stress estimates while relying on a robust solver with good scaling properties for large models. This aspect may be very appealing for safety considerations in a structural design context.

Another main advantage is the absence of user intervention while using the IPM. While contact solution algorithms and/or parameters can greatly influence calculation times or sometimes cause divergence, the IPM offers robust and efficient convergence behaviour with no user intervention and no algorithmic input parameter.

Finally, the present work considered a node-to-node discretization of contact conditions which requires matching meshes. However, the proposed approach does not rely on a 
specific discretization technique and could perfectly be extended to more advanced strategies involving non-matching meshes with a node-to-surface method for instance. Curved surfaces such as the bolt cores or plate holes can also be finely modelled using curved or isogeometric elements.

Further developments to include plastic constitutive relations which can be expressed as second order conic conditions as well are currently investigated. This method should be able to tackle such problems without using an incremental approach which can be hard to pilot using a classical Newton-Raphson algorithm and even surpass the problems faced when using non-conventional equilibrium elements. Although equilibrium elements are not widely used in industrial software, it offers the advantage of assessing the solution convergence by comparison with the primal displacement-based solution but also of computing a lower-bound solution and, therefore, a safer solution in terms of stress quantities than a displacement-based solution. Its use will therefore be even more interesting in the context of safety verifications including elasto-plastic behaviour and limit load computations. These aspects will be investigated in a future contribution.

Although the efficiency of IPM in the context of contact elastostatics has been illustrated, the main bottleneck that needs to be tackled is the linear solver of the Newton system. The use of direct solvers for really large-scale 3D problems becomes prohibitive and iterative solvers would certainly be more appropriate. Development of good preconditioners of IPM is however still a subject of current research. Another question concerns modelling choices of frictional contact. Due to its formulation relying on a convex optimization problem, the proposed IPM approach cannot take into account the real non-associative behaviour of Coulomb's frictional contact as such. Some strategies have nonetheless been already proposed to circumvent this aspect [36, 37, 58, 31] and this problem will definitely deserve further consideration.

\section{Appendix A Constitutive error estimator}

As stated in [56], many constitutive relations can be defined using a pair of convex dual functions $\varphi$ and $\varphi^{*}$ such that:

$$
\varphi_{(\varepsilon)}+\varphi_{(\sigma)}^{*}-\sigma: \varepsilon \geq 0
$$

This is no other than the Fenchel inequality for a pair of Fenchel conjugate functions. At any point in $\Omega$, the equality in the above equation is strictly equivalent to enforcing the constitutive relation at that point:

$$
\varphi_{(\varepsilon)}+\varphi_{(\boldsymbol{\sigma})}^{*}-\boldsymbol{\sigma}: \boldsymbol{\varepsilon}=0 \Leftrightarrow \boldsymbol{\sigma}=\frac{\partial \varphi}{\partial \boldsymbol{\varepsilon}} \text { and } \boldsymbol{\varepsilon}=\frac{\partial \varphi^{*}}{\partial \boldsymbol{\sigma}}
$$

Using this concept, we define a constitutive relation error including the same volume term defined in [56] and adding a surface term expressed over the contact boundary $\Gamma_{\boldsymbol{c}}$ as in 
[59, 60, 61]:

$$
\begin{aligned}
e_{(\boldsymbol{u}, \boldsymbol{\sigma})}^{2} & =e_{\Omega(\boldsymbol{u}, \boldsymbol{\sigma})}^{2}+e_{\Gamma_{\boldsymbol{c}}(\boldsymbol{u}, \boldsymbol{\sigma})}^{2} \\
e_{(\boldsymbol{u}, \boldsymbol{\sigma})}^{2} & =\int_{\Omega}\left(\varphi_{\left(\varepsilon_{(\boldsymbol{u})}\right)}+\varphi_{(\boldsymbol{\sigma})}^{*}-\boldsymbol{\sigma}: \boldsymbol{\varepsilon}_{(\boldsymbol{u})}\right) d \Omega \\
& +\int_{\Gamma_{\boldsymbol{c}}}\left(\mathbb{1}_{\mathcal{K}_{\mu}^{\circ}\left(\boldsymbol{g}_{(\boldsymbol{u})}\right)}+\mathbb{1}_{\mathcal{K}_{\mu}(\boldsymbol{\sigma} \cdot \boldsymbol{n})}-(\boldsymbol{\sigma} \cdot \boldsymbol{n}) \cdot \boldsymbol{g}_{(\boldsymbol{u})}\right) d \Gamma
\end{aligned}
$$

with $\mathbb{1}_{\mathcal{K}_{\mu}^{\circ}(\boldsymbol{g})}$ and $\mathbb{1}_{\mathcal{K}_{\mu}(\boldsymbol{\sigma} \boldsymbol{n})}$ being the indicator functions of the contact cones defined in section 2.1.3 such as :

$$
\mathbb{1}_{\mathcal{K}(\boldsymbol{x})}= \begin{cases}1 & \text { if } \boldsymbol{x} \in \mathcal{K} \\ +\infty & \text { otherwise }\end{cases}
$$

Indeed, for the convex cone $\mathcal{K}_{\mu}$, we have that the conjugate of the indicator function of the cone if no other than the indicator function of its polar cone i.e. $\left(\mathbb{1}_{\mathcal{K}_{\mu}}\right)^{*}=\mathbb{1}_{\mathcal{K}_{\mu}^{\circ}}$ and it can be easily shown that:

$$
\mathbb{1}_{\mathcal{K}_{\mu}^{\circ}(\boldsymbol{g})}+\mathbb{1}_{\mathcal{K}_{\mu}(\boldsymbol{\sigma} . \boldsymbol{n})}-(\boldsymbol{\sigma} \cdot \boldsymbol{n}) \cdot \boldsymbol{g}=0
$$

is equivalent to conditions (2.5), 2.6) and (2.8). The important property of this error functional is that it is always positive:

$$
e_{(\boldsymbol{u}, \boldsymbol{\sigma})}^{2} \geq 0 \quad \forall(\boldsymbol{u}, \boldsymbol{\sigma})
$$

In particular, it will be infinite if the contact variables do not satisfy the contact constitutive equations.

For the purpose of the present paper considering only elastic behaviour in the bulk domain, $\varphi_{(\varepsilon)}$ and $\varphi_{(\boldsymbol{\sigma})}^{*}$ coincide with Helmholtz's free energy and Gibbs's complementary energy in the case of linear elasticity:

$$
\varphi_{(\varepsilon)}=\frac{1}{2} \varepsilon: \mathbb{C}: \varepsilon \quad \text { and } \quad \varphi_{(\sigma)}^{*}=\frac{1}{2} \boldsymbol{\sigma}: \mathbb{C}^{-1}: \boldsymbol{\sigma}
$$

It can be easily shown that the error can be expressed as follows for any $\boldsymbol{\sigma} \in S A$ and $\boldsymbol{u} \in K A$ :

$$
e_{(\boldsymbol{u}, \boldsymbol{\sigma})}^{2}=\Pi_{p(\boldsymbol{u})}+\Pi_{c(\boldsymbol{\sigma})}
$$

with $\Pi_{p(\boldsymbol{u})}$ being the well-known total elastic potential energy (TPE) functional including a surface contact term:

$$
\Pi_{p(\boldsymbol{u})}=\int_{\Omega} \frac{1}{2} \boldsymbol{\varepsilon}_{(\boldsymbol{u})}: \mathbb{C}: \boldsymbol{\varepsilon}_{(\boldsymbol{u})} d \Omega+\int_{\Gamma_{\boldsymbol{c}}} \mathbb{1}_{\mathcal{K}_{\mu}^{\circ}\left(\boldsymbol{g}_{(\boldsymbol{u})}\right)} d \Gamma-\int_{\Omega} \boldsymbol{b} . \boldsymbol{u} d \Omega-\int_{\Gamma_{\boldsymbol{t}}} \boldsymbol{t}_{d} \cdot \boldsymbol{u} d \Gamma
$$

and $\Pi_{c(\boldsymbol{\sigma})}$ being the well-known total elastic complementary energy (TCPE) functional also 
including a surface contact term:

$$
\Pi_{c(\boldsymbol{\sigma})}=\int_{\Omega} \frac{1}{2} \boldsymbol{\sigma}: \mathbb{C}^{-1}: \boldsymbol{\sigma} d \Omega+\int_{\Gamma_{\boldsymbol{c}}} \mathbb{1}_{\mathcal{K}_{\mu}(\boldsymbol{\sigma} \cdot \boldsymbol{n})} d \Gamma-\int_{\Gamma_{\boldsymbol{u}}}(\boldsymbol{\sigma} \cdot \boldsymbol{n}) . \boldsymbol{u}_{d} d \Gamma-\int_{\Gamma_{\boldsymbol{c}}}(\boldsymbol{\sigma} \cdot \boldsymbol{n}) \cdot\left(g_{0} \boldsymbol{n}\right) d \Gamma
$$

The introduced error also possesses the important property to be zero if and only if the constitutive equations are satisfied. As a result, the solution $\left(\boldsymbol{u}^{*}, \boldsymbol{\sigma}^{*}\right)$ can be characterized by:

$$
\left(\boldsymbol{u}^{*}, \boldsymbol{\sigma}^{*}\right) \in K A \times S A\left\{\begin{array}{c}
\boldsymbol{\sigma}^{*}=\mathbb{C}: \boldsymbol{\varepsilon}_{\left(u^{*}\right)} \\
\left(\boldsymbol{g}_{\left(\boldsymbol{u}^{*}\right)}, \boldsymbol{\sigma}^{*} . \boldsymbol{n}\right) \in\left(\mathcal{K}_{\mu} \times \mathcal{K}_{\mu}^{\circ}\right)
\end{array} \Leftrightarrow e_{\left(\boldsymbol{u}^{*}, \boldsymbol{\sigma}^{*}\right)}^{2}=0\right.
$$

Owing to the introduced assumptions, a solution to the previous problem always exists. It has been demonstrated in [59, 60, 61] that the error estimator described above always gives an upper bound for the real error i.e. let $\left(\boldsymbol{u}^{*}, \boldsymbol{\sigma}^{*}\right)$ be a solution to the contact problem, and $\left(\boldsymbol{u}_{h}, \boldsymbol{\sigma}_{h}\right) \in K A \times S A$ be an admissible approximation, then:

$$
\frac{1}{2}\left\|\boldsymbol{\sigma}_{h}-\boldsymbol{\sigma}^{*}\right\|_{\mathbb{C}^{-1}, \Omega}^{2}+\frac{1}{2}\left\|\boldsymbol{\varepsilon}_{\left(u_{h}\right)}-\boldsymbol{\varepsilon}_{\left(u^{*}\right)}\right\|_{\mathbb{C}, \Omega}^{2}-\mu \int_{\Gamma_{\boldsymbol{c}}}\left(\boldsymbol{\sigma}_{h} \cdot \boldsymbol{n}-\boldsymbol{\sigma}^{*} \cdot \boldsymbol{n}\right)\left(\boldsymbol{g}_{\left(\boldsymbol{u}_{h}\right)}-\boldsymbol{g}_{\left(\boldsymbol{u}^{*}\right)}\right) d \Gamma \leq e_{\left(\boldsymbol{u}_{h}, \boldsymbol{\sigma}_{h}\right)}^{2}
$$

with $\|\boldsymbol{\varepsilon}\|_{\mathbb{C}, \Omega}$ and $\|\boldsymbol{\sigma}\|_{\mathbb{C}^{-1}, \Omega}$ being the traditional energy norms. Further information about posteriori error estimation for contact problems can be found in [62, 63, 64]

\section{Appendix B Quadratic second-order cone programming}

Let us consider the following quadratic second-order cone program:

$$
\begin{array}{clr}
\min _{\boldsymbol{x}, \boldsymbol{x}_{i}} & \frac{1}{2} \boldsymbol{x}^{T} \boldsymbol{P} \boldsymbol{x}+\boldsymbol{c}^{T} \boldsymbol{x}+\sum_{i=1}^{m} \boldsymbol{c}_{i}^{T} \boldsymbol{x}_{i} \\
\text { s.t. } & \boldsymbol{A} \boldsymbol{x}=\boldsymbol{b} \\
& \boldsymbol{A}_{i} \boldsymbol{x}-\boldsymbol{x}_{i}=\boldsymbol{b}_{i} \quad i=1, \ldots, m \\
& \boldsymbol{x}_{i} \in \mathcal{K}_{i} & i=1, \ldots, m
\end{array}
$$

where $\boldsymbol{P}$ is a symmetric positive definite matrix and $\mathcal{K}_{i}, i=1, \ldots, m$ are different types of second-order self-dual cones such as:

- the positive orthant: $\mathbb{R}_{+}^{n_{i}}=\left\{\boldsymbol{z} \in \mathbb{R}^{n_{i}}\right.$ s.t. $\left.z_{j} \geq 0 \quad \forall j=1, \ldots, n_{i}\right\}$

- the Lorentz second-order cone:

$$
\mathcal{Q}^{n_{i}}=\left\{\mathbf{z}=\left(z^{0}, \overline{\mathbf{z}}\right) \in \mathbb{R} \times \mathbb{R}^{n_{i}-1} \text { s.t. } z^{0} \geq\|\overline{\mathbf{z}}\|\right\}
$$

- the rotated Lorentz second-order cone:

$$
\mathcal{Q}_{r}^{n_{i}}=\left\{\mathbf{z}=\left(z^{0}, z^{1}, \tilde{\mathbf{z}}\right) \in \mathbb{R} \times \mathbb{R} \times \mathbb{R}^{n_{i}-2} \text { s.t. } 2 z^{1} z^{0} \geq\|\tilde{\mathbf{z}}\|^{2}\right\}
$$


These cones all possess the important property of being self-dual: $\mathcal{K}_{i}^{*}=\mathcal{K}_{i}$.

Most standard SOCP program formats assumes no quadratic objective term, but the previous problem can be always reformulated as a problem involving a linear objective function only by introducing auxiliary variables and additional conic constraints [52, 20, 22]. However, in our implementation, the quadratic term will be kept as such in the objective function.

We can construct the Lagrangian $\mathcal{L}$ of the previous program as follows:

$$
\begin{aligned}
\mathcal{L}\left(\boldsymbol{x}, \boldsymbol{x}_{i}, \boldsymbol{\lambda}, \boldsymbol{\nu}_{i}, \boldsymbol{s}_{i}\right)= & \frac{1}{2} \boldsymbol{x}^{T} \boldsymbol{P} \boldsymbol{x}+\left(\boldsymbol{c}+\boldsymbol{A}^{T} \boldsymbol{\lambda}+\sum_{i=1}^{m} \boldsymbol{A}_{i}^{T} \boldsymbol{\nu}_{i}\right)^{T} \boldsymbol{x} \\
& +\sum_{i=1}^{m}\left(\boldsymbol{c}_{i}-\boldsymbol{\nu}_{i}-\boldsymbol{s}_{i}\right)^{T} \boldsymbol{x}_{i}-\boldsymbol{\lambda}^{T} \boldsymbol{b}-\sum_{i=1}^{m} \boldsymbol{\nu}_{i}^{T} \boldsymbol{b}_{i}
\end{aligned}
$$

Let $\star_{I}^{T}=\left\{\ldots, \star_{i}^{T}, \ldots\right\}$ be the seperate concatenation of each vector indexed by $i, \boldsymbol{A}_{I}=$ $\boldsymbol{d i a g}\left(\ldots, \boldsymbol{A}_{i}, \ldots\right)$ and $\mathcal{K}$ the tensorial product of the $\mathcal{K}_{i}$ cones i.e. $\boldsymbol{x} \in \mathcal{K} \Leftrightarrow \boldsymbol{x}_{i} \in \mathcal{K}_{i}, \forall i=$ $1, \cdots, n$. Problem (B.1) becomes:

$$
\begin{array}{cl}
\min _{\boldsymbol{x}, \boldsymbol{x}_{I}} & \frac{1}{2} \boldsymbol{x}^{T} \boldsymbol{P} \boldsymbol{x}+\boldsymbol{c}^{T} \boldsymbol{x}+\boldsymbol{c}_{I}^{T} \boldsymbol{x}_{I} \\
\text { s.t. } & \boldsymbol{A} \boldsymbol{x}=\boldsymbol{b} \\
& \boldsymbol{A}_{I} \boldsymbol{x}-\boldsymbol{x}_{I}=\boldsymbol{b}_{I} \\
& \boldsymbol{x}_{I} \in \mathcal{K}
\end{array}
$$

and the Lagrangian can be expressed as:

$$
\mathcal{L}\left(\boldsymbol{x}, \boldsymbol{x}_{I}, \boldsymbol{\lambda}, \boldsymbol{\nu}_{I}, \boldsymbol{s}_{I}\right)=\frac{1}{2} \boldsymbol{x}^{T} \boldsymbol{P} \boldsymbol{x}+\left(\boldsymbol{c}+\boldsymbol{A}^{T} \boldsymbol{\lambda}+\boldsymbol{A}_{I}^{T} \boldsymbol{\nu}_{I}\right)^{T} \boldsymbol{x}+\left(\boldsymbol{c}_{I}-\boldsymbol{\nu}_{I}-\boldsymbol{s}_{I}\right)^{T} \boldsymbol{x}_{I}-\boldsymbol{\lambda}^{T} \boldsymbol{b}-\boldsymbol{\nu}_{I}^{T} \boldsymbol{b}_{I}
$$

The corresponding KKT optimality conditions are:

$$
\begin{array}{r}
\partial_{\boldsymbol{x}} \mathcal{L}=\boldsymbol{P} \boldsymbol{x}+\boldsymbol{c}+\boldsymbol{A}^{T} \boldsymbol{\lambda}+\boldsymbol{A}_{I}^{T} \boldsymbol{\nu}_{I}=0 \\
\partial_{\boldsymbol{x}_{I}} \mathcal{L}=\boldsymbol{c}_{I}-\boldsymbol{\nu}_{I}-\boldsymbol{s}_{I}=0 \\
\partial_{\boldsymbol{\lambda}} \mathcal{L}=\boldsymbol{A} \boldsymbol{x}-\boldsymbol{b}=0 \\
\partial_{\boldsymbol{\nu}_{I}} \mathcal{L}=\boldsymbol{A}_{I} \boldsymbol{x}-\boldsymbol{x}_{I}-\boldsymbol{b}_{I}=0 \\
\boldsymbol{x}_{I} \in \mathcal{K}, \boldsymbol{s}_{I} \in \mathcal{K}^{*}, \boldsymbol{x}_{I}^{T} \boldsymbol{s}_{I}=0
\end{array}
$$

where $\mathcal{K}^{*}$ is the tensorial product of the dual cones $\mathcal{K}_{i}^{*}$.

\section{Appendix C Implementation of the primal-dual interior-point method}

\section{C.1 The perturbed KKT system}

At each iteration $k$, a Newton step on the perturbed KKT system (5.3) is computed towards the central path for a fixed value of $\eta^{k}$. The solution is updated after a step-length 
calculation and the barrier parameter is reduced by some amount. The process is repeated until the residuals fall under a certain tolerance. This will result in a series of iterates $\boldsymbol{z}^{k}=\left(\boldsymbol{x}^{k}, \boldsymbol{x}_{I}^{k}, \boldsymbol{\lambda}^{k}, \boldsymbol{\nu}_{I}^{k}, \boldsymbol{s}_{I}^{k}\right)$ which remain feasible regarding the conic constraints.

Given an iteration $k$ at which a point $\boldsymbol{z}^{k}$ satisfying the conic constraints is known and a value for the barrier parameter $\eta^{k}$ has been chosen, the next iterate is calculated by computing a new point near the central path. This is obtained by performing one iteration of the Newton method when linearising the residuals equation such as $\boldsymbol{r}^{k+1}(\boldsymbol{z})=$ $\boldsymbol{r}^{k}(\boldsymbol{z})+\boldsymbol{r}^{\prime k}(\boldsymbol{z}) \cdot \boldsymbol{\Delta} \boldsymbol{z}=0$ thus solving the system $\boldsymbol{D}^{k} \cdot \boldsymbol{\Delta} \boldsymbol{z}=-\boldsymbol{r}^{k}(\boldsymbol{z})$ with $\boldsymbol{D}^{k}=\boldsymbol{r}^{\prime k}(\boldsymbol{z})$. Hence, a descent direction $\boldsymbol{\Delta} \boldsymbol{z}=\left(\boldsymbol{\Delta x}, \boldsymbol{\Delta} \boldsymbol{x}_{I}, \boldsymbol{\Delta} \boldsymbol{\lambda}, \boldsymbol{\Delta} \boldsymbol{\nu}_{I}, \boldsymbol{\Delta} \boldsymbol{s}_{I}\right)$ is computed by linearising the perturbed KKT system around $\boldsymbol{z}^{k}$ as follows:

$$
\boldsymbol{D}^{k} \boldsymbol{\Delta} \boldsymbol{z}=\left[\begin{array}{ccccc}
\boldsymbol{P} & \mathbf{0} & \boldsymbol{A}^{T} & \boldsymbol{A}_{I}^{T} & \mathbf{0} \\
\mathbf{0} & \mathbf{0} & \mathbf{0} & \boldsymbol{I} & \boldsymbol{I} \\
\boldsymbol{A} & \mathbf{0} & \mathbf{0} & \mathbf{0} & \mathbf{0} \\
\boldsymbol{A}_{I} & -\boldsymbol{I} & \mathbf{0} & \mathbf{0} & \mathbf{0} \\
\mathbf{0} & \boldsymbol{S}_{I}^{k} & \mathbf{0} & \mathbf{0} & \boldsymbol{X}_{I}^{k}
\end{array}\right]\left\{\begin{array}{c}
\boldsymbol{\Delta} \boldsymbol{x} \\
\boldsymbol{\Delta} \boldsymbol{x}_{I} \\
\boldsymbol{\Delta} \boldsymbol{\lambda} \\
\boldsymbol{\Delta} \boldsymbol{\nu}_{I} \\
\boldsymbol{\Delta} \boldsymbol{s}_{I}
\end{array}\right\}=\left\{\begin{array}{c}
-r_{d}^{k} \\
-r_{d I}^{k} \\
-r_{p}^{k} \\
-r_{p I}^{k} \\
-r_{c}^{k}
\end{array}\right\}=-\boldsymbol{r}^{k}(\boldsymbol{z})
$$

where $\boldsymbol{X}_{I}^{k}=\boldsymbol{d i a g}\left(\ldots, \boldsymbol{m a t}\left(\boldsymbol{x}_{i}^{k}\right), \ldots\right)$ and $\boldsymbol{S}_{I}^{k}=\boldsymbol{d i a g}\left(\ldots, \boldsymbol{m a t}\left(\boldsymbol{s}_{i}^{k}\right), \ldots\right)$ and the vector $\boldsymbol{r}^{k}$ corresponding to the vector of residuals:

$$
\begin{aligned}
r_{d}^{k} & =\boldsymbol{P} \boldsymbol{x}^{k}+\boldsymbol{A}^{T} \boldsymbol{\lambda}^{k}+\boldsymbol{A}_{I}^{T} \boldsymbol{\nu}_{I}^{k}+\boldsymbol{c} \\
r_{d I}^{k} & =\boldsymbol{c}_{I}-\boldsymbol{\nu}_{I}^{k}-\boldsymbol{s}_{I}^{k} \\
r_{p}^{k} & =\boldsymbol{A} \boldsymbol{x}^{k}-\boldsymbol{b} \\
r_{p I}^{k} & =\boldsymbol{A}_{I} \boldsymbol{x}^{k}-\boldsymbol{x}_{I}^{k}-\boldsymbol{b}_{I} \\
r_{c}^{k} & =\eta^{k} \boldsymbol{e}_{I}-\boldsymbol{X}_{I}^{k} \boldsymbol{S}_{I}^{k} \boldsymbol{e}_{I}
\end{aligned}
$$

Details about the linearisation of the complementarity condition $5.3 \mathrm{e}$ along with the definition of matrices $\boldsymbol{m a t}\left(\boldsymbol{x}_{i}\right)$ and $\boldsymbol{m a t}\left(\boldsymbol{s}_{i}\right)$ involved can be found in appendix D.

\section{C.2 Step-length calculation}

The next iterate $\boldsymbol{z}^{k+1}$ is obtained by performing a line-search on the maximum allowable step length $\alpha$ in direction $\boldsymbol{\Delta} \boldsymbol{z}$, i.e. $\boldsymbol{z}^{k+1}=\boldsymbol{z}^{k}+\alpha \boldsymbol{\Delta} \boldsymbol{z}$ with $\alpha \in[0,1]$. This step should be chosen such that the iterate still satisfies the conic constraints. One can use a simple line-search procedure but a fraction-to-boundary rule is used to limit its value to avoid that the next iterate will lie exactly on the boundary of the feasible domain. Practically, $\alpha$ is chosen such as $\alpha \leq \alpha_{\max }<1$ with $\alpha_{\max }=0.995$ typically. The primal and dual residuals are then reduced by a $(1-\alpha)$ ratio. Following the central path which is located far from the feasibility boundaries will allow for large steps to be taken. The IPM thus shows all its efficiency compared to a traditional descent method along the gradient of the objective function where the boundary of the feasible region will be reached in the early stages of the algorithm, leading to small progress when following the boundary. 


\section{C.3 Choice of the barrier parameter}

The choice of the barrier parameter $\eta$ at the beginning of each step influences the whole performance of the IP algorithm. This choice will define the type of each step, whether a "centering" step is made in which the next iterate will be closer to the central path, or a "descent" step in which the complementarity gap is reduced but maybe to the detriment of going away from the central path. We recall that in order to solve the perturbed KKT system and converge to the optimal solution, the barrier parameter $\eta$ should be driven to 0 . At the end of an iteration $(k+1)$, a new feasible point $\boldsymbol{z}^{k+1}$ has been calculated, and a typical choice for the new barrier parameter $\eta^{k+1}$ makes use of the value of the complementarity gap $g_{i}^{k+1}=\boldsymbol{s}_{i}^{k+1} \cdot \boldsymbol{x}_{i}^{k+1}$ for each cone:

$$
\eta^{k+1}=\gamma\left(\frac{1}{m} \sum_{i=1}^{m} g_{i}^{k+1}\right)=\gamma \bar{g}^{k+1}
$$

$m$ being the total number of cones. The barrier parameter $\eta^{k+1}$ is therefore proportional to the average complementarity gap via a scalar $\gamma \in[0,1]$. This parameter can be chosen in an adaptive matter as will be explained in section C.5.1.

\section{C.4 Initialization and convergence criteria}

All primal non-conic variables i.e. $\boldsymbol{x}$ are initially taken equal to 0 , whereas the conic variables and their dual are taken as $\boldsymbol{x}_{i}=\left(x_{i}^{0}, \overline{\boldsymbol{x}}_{i}\right)=(1, \mathbf{0})$. This choice ensures that we have a starting point that is feasible with the conic constraints. The initial complementarity gap is then equal to 1 everywhere. As the residual vectors and the complementarity gap are both reduced at the same rate, the same stopping criterion is used. The iterative process is stopped when the complementarity gap and the residual norms fall bellow a given tolerance, here fixed to $10^{-8}$. This point will be considered as an optimal solution for the non-perturbed KKT system. The algorithm will also be stopped if the number of iterations exceeds 50, and then the algorithm will have failed to find an optimal solution. In practice, the average number of iterations was always found between 15 and 25 at most.

\section{C.5 Some advanced aspects of the IPM}

\section{C.5.1 Predictor-corrector scheme and adaptive choice of the barrier parameter}

One of the major improvements in IPM was made by Mehrotra [43] who proposed an adaptive scheme for the choice of the barrier parameter $\eta$. As explained before, the choice of the centering parameter $\gamma$ will determine the trade-off between improving centering and reducing the complementarity gap. The idea behind the method is to take advantage of the factorisation of the KKT matrix $\boldsymbol{D}$ which takes most of the computing time. Once done, it is quite inexpensive to solve a second linear system using the same matrix but using different residuals. Hence the idea is to perform two calculation steps as follows:

1. the first step, called affine step, is calculated using $\eta=0$, and its solution is denoted $\boldsymbol{\Delta} \boldsymbol{z}^{a}$. The maximum step length for this step is noted $\alpha_{\max }^{a}$. This step amounts to solving the unperturbed KKT system; 
2. The centering parameter is then chosen using the heuristic presented in [21]:

$$
\gamma=\left(1-\alpha_{\max }^{a}\right) \min \left\{0.5 ;\left(1-\alpha_{\max }^{a}\right)^{2}\right\}
$$

If $\alpha_{\max }^{a}$ is large, only a little centering will be necessary (the iterate being far from the boundary). If, on the contrary, $\alpha_{\max }^{a}$ is small, the feasible region boundaries have been reached and centering will be restored by choosing a large value for $\gamma$, driving the next iterate closer to the central path;

3. the final step direction $\boldsymbol{\Delta} \boldsymbol{z}$ is then computed using the value of $\eta^{k}$ calculated using the equation (C.3) and a reduced residuals vector depending on $\gamma$, ensuring that residuals and complementarity gaps are reduced at the same rate.

Further details about the choice of the parameters and the reduction of the residuals can be found in [55, 53, 43].

\section{C.5.2 Reduced linear system}

It is possible to reduce the system (C.1) to a smaller system by eliminating the conic primal and dual variables $\Delta \boldsymbol{x}_{I}$ and $\Delta \boldsymbol{s}_{I}$ and the corresponding Lagrange variables $\Delta \boldsymbol{\nu}_{I}$. Unfortunately, the system will generally be non-symmetric (except for linear programming). A rescaling of the complementarity conditions (5.3e) using the approach proposed by Nesterov and Todd [44] enables to obtain a symmetric reduced system of the following form:

$$
\left[\begin{array}{cc}
\boldsymbol{P}+\boldsymbol{A}_{I}^{T} \boldsymbol{F}_{I}^{2} \boldsymbol{A}_{I} & \boldsymbol{A}^{T} \\
\boldsymbol{A} & \mathbf{0}
\end{array}\right]\left\{\begin{array}{c}
\boldsymbol{\Delta} \boldsymbol{x} \\
\boldsymbol{\Delta} \boldsymbol{\lambda}
\end{array}\right\}=\left\{\begin{array}{c}
-r_{d}^{\prime k} \\
-r_{p}^{k}
\end{array}\right\}
$$

where:

$$
\begin{gathered}
r_{d}^{\prime k}=r_{d}^{k}+\boldsymbol{A}_{I}^{T} \boldsymbol{F}_{I} \boldsymbol{V}_{I}^{-1} r_{c}^{k}+\boldsymbol{A}_{I}^{T} \boldsymbol{F}_{I}^{2} r_{p I}^{k} \\
r_{p}^{k}=\boldsymbol{A} \boldsymbol{x}^{k}-\boldsymbol{b}
\end{gathered}
$$

The general details for the system's reduction and symmetric scaling can be found in appendix D.

The resulting system is still sparse but is now much smaller and symmetric semi-definite positive. Since the matrices $\boldsymbol{A}_{i}$ will only affect variables included in cones and its sparsity pattern is block-diagonal, it does not change the global sparsity pattern of matrix $\boldsymbol{P}$. The other variable increments are simply obtained by back-substitution.

Since the matrices $\boldsymbol{F}_{i}$ and their global concatenation $\boldsymbol{F}_{I}$ depend on each iterate, the solved system is very similar to those arising in Newton-Raphson algorithms with a tangent matrix and Lagrange multipliers to enforce boundary conditions. In this work, system (C.5) is solved using MKL Pardiso direct solver [65, 66, 67].

\section{Appendix D About the complementarity condition for second-order cone}

General results concerning Jordan algebra over the Lorentz second-order cone and the Nesterov-Todd scaling are given here. For a vector $\boldsymbol{v}=\left(v^{0}, \overline{\boldsymbol{v}}\right) \in \mathcal{Q}^{m+1}$, we define: 


$$
\begin{aligned}
\boldsymbol{m a t}(\boldsymbol{v}) & =\left[\begin{array}{cc}
v^{0} & \overline{\boldsymbol{v}}^{T} \\
\overline{\boldsymbol{v}} & v^{0} \boldsymbol{I}_{m}
\end{array}\right] \in \mathbb{R}^{(m+1) \times(m+1)} \\
\operatorname{det}(\boldsymbol{v}) & =\operatorname{det}(\boldsymbol{m a t}(\boldsymbol{v}))=\left(v^{0}\right)^{2}-\|\overline{\boldsymbol{v}}\|^{2} \\
\boldsymbol{Q} & =\boldsymbol{d i a g}\left(1,-\boldsymbol{I}_{m}\right) \\
\hat{\boldsymbol{v}} & =\boldsymbol{Q} \boldsymbol{v}=\left(v^{0},-\overline{\boldsymbol{v}}\right) \\
(\boldsymbol{m a t}(\boldsymbol{v}))^{-1} & =\frac{1}{\operatorname{det}(\boldsymbol{v})}\left[\begin{array}{cc}
v^{0} & -\overline{\boldsymbol{v}}^{T} \\
-\overline{\boldsymbol{v}} & \frac{\operatorname{det}(\boldsymbol{v}) \boldsymbol{I}_{m}+\overline{\boldsymbol{v} \boldsymbol{v}^{T}}}{v^{0}}
\end{array}\right]
\end{aligned}
$$

The last equation is valid only if $\boldsymbol{v}$ is located strictly inside $\mathcal{Q}^{m+1}$, i.e. $\boldsymbol{v} \in \operatorname{int}\left(\mathcal{Q}^{m+1}\right)$, which is equivalent to $\operatorname{det}(\boldsymbol{v})>0$.

Let $\boldsymbol{X}=\boldsymbol{m a t}(\boldsymbol{x})$ and $\boldsymbol{S}=\boldsymbol{\operatorname { m a t }}(\boldsymbol{s})$, the complementarity condition (5.4) can be rewritten as:

$$
\boldsymbol{x} \circ \boldsymbol{s}=\left\{\begin{array}{c}
x^{0} s^{0}+\overline{\boldsymbol{x}}^{T} \overline{\boldsymbol{s}} \\
x^{0} \overline{\boldsymbol{s}}+s^{0} \overline{\boldsymbol{x}}
\end{array}\right\}=\boldsymbol{X} \boldsymbol{s}=\boldsymbol{S} \boldsymbol{x}=\boldsymbol{X} \boldsymbol{S e}
$$

with $\boldsymbol{e}=(1, \mathbf{0})$. The linearised form can then be written as:

$$
(\boldsymbol{x} \circ \boldsymbol{s})^{(k+1)} \simeq \boldsymbol{X}^{k} \boldsymbol{S}^{k} \boldsymbol{e}+\boldsymbol{X}^{k} \boldsymbol{\Delta} \boldsymbol{s}+\boldsymbol{S}^{k} \boldsymbol{\Delta} \boldsymbol{x}
$$

It can be shown that for $\boldsymbol{x}, \boldsymbol{s} \in \operatorname{int}\left(\mathcal{Q}^{m+1}\right)$, there exists a unique matrix $\boldsymbol{F}$, depending on $\boldsymbol{x}$ and $\boldsymbol{s}$, such that

$$
\boldsymbol{F} \boldsymbol{x}=\tilde{\boldsymbol{x}}=\tilde{\boldsymbol{s}}=\boldsymbol{F}^{-1} \boldsymbol{S} \text { and } \boldsymbol{x} \circ \boldsymbol{s}=\tilde{\boldsymbol{x}} \circ \tilde{\boldsymbol{s}}
$$

Let $\boldsymbol{V}=\boldsymbol{m a t}(\tilde{\boldsymbol{x}})=\boldsymbol{m a t}(\tilde{\boldsymbol{s}})$ the associated matrix for the scaled point $\tilde{\boldsymbol{x}}=\tilde{\boldsymbol{s}}$. Using this symmetrical Nesterov-Todd scaling, the linearised complementarity condition can be rewritten as

$$
(\boldsymbol{x} \circ \boldsymbol{s})^{(k+1)} \simeq(\boldsymbol{x} \circ \boldsymbol{s})^{(k)}+\boldsymbol{V} \boldsymbol{F}^{-1} \boldsymbol{\Delta} \boldsymbol{s}+\boldsymbol{V} \boldsymbol{F} \boldsymbol{\Delta} \boldsymbol{x}
$$

Further details and the expression for matrix $\boldsymbol{F}$ can be found in [52, 22, 21, 44].

The KKT system (C.1) can then be efficiently reduced. Using the relation : $\boldsymbol{\nu}_{I}=\boldsymbol{c}_{I}-\boldsymbol{s}_{I}$, the $\boldsymbol{\Delta} \boldsymbol{\nu}_{I}$ variable can first be eliminated along with the second row. The first residual is then changed into:

$$
r_{d}^{k}=\boldsymbol{P} \boldsymbol{x}^{k}+\boldsymbol{A}^{T} \boldsymbol{\lambda}^{k}-\boldsymbol{A}_{I}^{T} \boldsymbol{s}_{I}^{k}+\boldsymbol{A}_{I}^{T} \boldsymbol{c}_{I}+\boldsymbol{c}
$$

and the KKT system now becomes:

$$
\boldsymbol{D}^{k} \boldsymbol{\Delta} \boldsymbol{z}=\left[\begin{array}{cccc}
\boldsymbol{P} & \boldsymbol{A}^{T} & -\boldsymbol{A}_{I}^{T} & \mathbf{0} \\
\boldsymbol{A} & \mathbf{0} & \mathbf{0} & \mathbf{0} \\
\boldsymbol{A}_{I} & -\boldsymbol{I} & \mathbf{0} & \mathbf{0} \\
\mathbf{0} & \left(\boldsymbol{V}_{I} \boldsymbol{F}_{I}\right)^{k} & \mathbf{0} & \left(\boldsymbol{V}_{I} \boldsymbol{F}_{I}^{-1}\right)^{k}
\end{array}\right]\left\{\begin{array}{c}
\boldsymbol{\Delta} \boldsymbol{x} \\
\boldsymbol{\Delta} \boldsymbol{x}_{I} \\
\boldsymbol{\Delta} \boldsymbol{\lambda} \\
\boldsymbol{\Delta} \boldsymbol{s}_{I}
\end{array}\right\}=\left\{\begin{array}{c}
-r_{d}^{k} \\
-r_{p}^{k} \\
-r_{p I}^{k} \\
-r_{c}^{k}
\end{array}\right\}=-\boldsymbol{r}^{k}(\boldsymbol{z})
$$


where $\boldsymbol{V}_{I}^{k}=\boldsymbol{d} \boldsymbol{d i a g}\left(\ldots, \boldsymbol{V}_{i}^{k}, \ldots\right)$ and $\boldsymbol{F}_{I}^{k}=\boldsymbol{d i a g}\left(\ldots, \boldsymbol{F}_{i}^{k}, \ldots\right)$. Eliminating $\boldsymbol{\Delta} \boldsymbol{x}_{I}$ and $\boldsymbol{\Delta} \boldsymbol{s}_{I}$ using

$$
\begin{gathered}
\boldsymbol{\Delta} \boldsymbol{s}_{I}=-\boldsymbol{F}_{I} \boldsymbol{V}_{I}^{-1} r_{c}^{k}-\boldsymbol{F}_{I}^{2} \boldsymbol{\Delta} \boldsymbol{x}_{I} \\
\boldsymbol{\Delta} \boldsymbol{x}_{I}=\boldsymbol{A}_{I} \boldsymbol{\Delta} \boldsymbol{x}+r_{p I}^{k}
\end{gathered}
$$

one finally obtains the reduced system (C.5. 


\section{References}

\section{References}

[1] N. Kikuchi, J. Oden, Contact problems in elasticity : a study of variational inequalities and finite element methods, Studies in Applied and Numerical Mathematics, Society for Industrial and Applied Mathematics, 1988. doi:10.1137/1.9781611970845.

[2] M. R. Hestenes, Multiplier and gradient methods, Journal of optimization theory and applications 4 (5) (1969) 303-320.

[3] M. J. Powell, A method for nonlinear constraints in minimization problems, Academic Press, 1969.

[4] P. Wriggers, G. Zavarise, Application of augmented Lagrangian techniques for nonlinear constitutive laws in contact interfaces, Communications in Numerical Methods in Engineering 9 (10) (1993) 815-824. doi:10.1002/cnm.1640091005.

[5] M. Fortin, R. Glowinski, Augmented Lagrangian methods: applications to the numerical solution of boundary-value problems, Elsevier, 2000.

[6] J. C. Simo, T. Laursen, An augmented lagrangian treatment of contact problems involving friction, Computers \& Structures 42 (1) (1992) 97-116.

[7] T. Laursen, J. Simo, Algorithmic symmetrization of coulomb frictional problems using augmented lagrangians, Computer methods in applied mechanics and engineering 108 (1-2) (1993) 133-146.

[8] P. Alart, A. Curnier, A mixed formulation for frictional contact problems prone to newton like solution methods, Computer methods in applied mechanics and engineering 92 (3) (1991) 353-375.

[9] P. Alart, Méthode de Newton généralisée en mécanique du contact, Journal de Mathématiques Pures et Appliquées 76 (1) (1997) 83-108. doi:10.1016/S0021-7824(97) 89946-1.

[10] S. Hüeber, B. I. Wohlmuth, A primal-dual active set strategy for non-linear multibody contact problems, Computer Methods in Applied Mechanics and Engineering 194 (2729) (2005) 3147-3166.

[11] M. Hintermüller, K. Ito, K. Kunisch, The primal-dual active set strategy as a semismooth Newton method, SIAM Journal on Optimization 13 (3) (2002) 865-888. doi:10.1137/S1052623401383558.

[12] A. Popp, W. Wall, Dual mortar methods for computational contact mechanicsoverview and recent developments, GAMM-Mitteilungen 37 (1) (2014) 66-84. 
[13] Y. Kanno, Nonsmooth mechanics and convex optimization, CRC Press, Boca Raton, FL, 2011.

[14] Y. Kanno, J. A. C. Martins, A. Pinto da Costa, Three-dimensional quasi-static frictional contact by using second-order cone linear complementarity problem, International Journal for Numerical Methods in Engineering 65 (1) (2006) 62-83. doi:10.1002/nme.1493.

[15] H. Zhang, J. Li, S. Pan, New second-order cone linear complementarity formulation and semi-smooth newton algorithm for finite element analysis of $3 \mathrm{~d}$ frictional contact problem, Computer Methods in Applied Mechanics and Engineering 200 (1-4) (2011) $77-88$.

[16] A. Klarbring, A mathematical programming approach to three-dimensional contact problems with friction, Computer Methods in Applied Mechanics and Engineering 58 (2) (1986) 175-200.

[17] D. E. Stewart, J. C. Trinkle, An implicit time-stepping scheme for rigid body dynamics with inelastic collisions and Coulomb friction, International Journal for Numerical Methods in Engineering 39 (15) (1996) 2673-2691.

[18] M. Hjiaj, Z.-Q. Feng, G. De Saxcé, Z. Mróz, Three-dimensional finite element computations for frictional contact problems with non-associated sliding rule, International Journal for Numerical Methods in Engineering 60 (12) (2004) 2045-2076.

[19] P. Joli, Z.-Q. Feng, Uzawa and Newton algorithms to solve frictional contact problems within the bi-potential framework, International Journal for Numerical Methods in Engineering 73 (3) (2008) 317-330. doi:10.1002/nme.2073.

[20] M. S. Lobo, L. Vandenberghe, S. Boyd, H. Lebret, Applications of second-order cone programming, Linear Algebra and its Applications 284 (1-3) (1998) 193-228. doi: $10.1016 /$ S0024-3795(98) 10032-0.

[21] E. Andersen, C. Roos, T. Terlaky, On implementing a primal-dual interior-point method for conic quadratic optimization, Mathematical Programming 95 (2) (2003) 249-277. doi:10.1007/s10107-002-0349-3.

[22] S. P. Boyd, L. Vandenberghe, Convex optimization, Cambridge University Press, Cambridge, UK ; New York, 2004.

[23] M. Wright, The interior-point revolution in optimization: history, recent developments, and lasting consequences, Bulletin of the American mathematical society 42 (1) (2005) 39-56.

[24] A. Makrodimopoulos, C. M. Martin, Lower bound limit analysis of cohesive-frictional materials using second-order cone programming, International Journal for Numerical Methods in Engineering 66 (4) (2006) 604-634. doi:10.1002/nme.1567. 
[25] A. Makrodimopoulos, C. M. Martin, Upper bound limit analysis using simplex strain elements and second-order cone programming, International Journal for Numerical and Analytical Methods in Geomechanics 31 (6) (2007) 835-865. doi:10.1002/nag. 567.

[26] A. V. Lyamin, S. W. Sloan, Lower bound limit analysis using non-linear programming, International Journal for Numerical Methods in Engineering 55 (5) (2002) 573-611.

[27] A. V. Lyamin, S. W. Sloan, Upper bound limit analysis using linear finite elements and non-linear programming, International Journal for Numerical and Analytical Methods in Geomechanics 26 (2) (2002) 181-216.

[28] J. Bleyer, M. Maillard, P. De Buhan, P. Coussot, Efficient numerical computations of yield stress fluid flows using second-order cone programming, Computer Methods in Applied Mechanics and Engineering 283 (2015) 599-614.

[29] J. Bleyer, Advances in the simulation of viscoplastic fluid flows using interior-point methods, Computer Methods in Applied Mechanics and Engineering 330 (2018) 368394.

[30] K. Krabbenhoft, A. Lyamin, J. Huang, M. Vicente da Silva, Granular contact dynamics using mathematical programming methods, Computers and Geotechnics 43 (2012) 165-176. doi:10.1016/j.compgeo.2012.02.006

[31] J. Kleinert, B. Simeon, M. Obermayr, An inexact interior point method for the largescale simulation of granular material, Computer Methods in Applied Mechanics and Engineering 278 (2014) 567-598.

[32] P. W. Christensen, A. Klarbring, J. S. Pang, N. Strömberg, Formulation and comparison of algorithms for frictional contact problems, International Journal for Numerical Methods in Engineering 42 (1) (1998) 145-173. doi:10.1002/(SICI) 1097-0207(19980515) 42:1<145: :AID-NME358>3.0.CO;2-L.

[33] G. Tanoh, Y. Renard, D. Noll, Computational experience with an interior point algorithm for large scale contact probles, Optimization Online 10 (2) (2004) 18.

[34] D. Mangoni, A. Tasora, R. Garziera, A primal-dual predictor-corrector interior point method for non-smooth contact dynamics, Computer Methods in Applied Mechanics and Engineering 330 (2018) 351-367.

[35] I. Temizer, M. Abdalla, Z. Gürdal, An interior point method for isogeometric contact, Computer Methods in Applied Mechanics and Engineering 276 (2014) 589-611.

[36] M. Gilbert, C. Casapulla, H. Ahmed, Limit analysis of masonry block structures with non-associative frictional joints using linear programming, Computers \& structures $84(13-14)$ (2006) 873-887. 
[37] K. Krabbenhoft, M. Karim, A. Lyamin, S. Sloan, Associated computational plasticity schemes for nonassociated frictional materials, International Journal for Numerical Methods in Engineering 90 (9) (2012) 1089-1117.

[38] O. C. Zienkiewicz, R. L. Taylor, The finite element method, 5th Edition, ButterworthHeinemann, Oxford ; Boston, 2000.

[39] B. Fraeijs de Veubeke, Diffusion des inconnues hyperstatiques dans les voilures à longerons couplés, Vol. 24, Hayez, 1951.

[40] J. de Almeida, J. de Freitas, Alternative approach to the formulation of hybrid equilibrium finite elements, Computers \& Structures 40 (4) (1991) 1043-1047. doi: 10.1016/0045-7949(91) 90336-K.

[41] J. P. M. De Almeida, O. J. B. A. Pereira, A set of hybrid equilibrium finite elements models for the analysis of three dimensional solids, International Journal for Numerical Methods in Engineering 39 (16) (1996) 2789-2802. doi:10.1002/(SICI) 1097-0207(19960830) 39:16<2789: :AID-NME976>3.0.CO;2-J.

[42] J. P. M. de Almeida, E. A. Maunder, Equilibrium finite element formulations, John Wiley \& Sons, Ltd, Chichester, UK, 2017. doi:10.1002/9781118925782.

[43] S. Mehrotra, On the implementation of a primal-dual interior point method, SIAM Journal on Optimization 2 (4) (1992) 575-601. doi:10.1137/0802028.

[44] Y. E. Nesterov, M. J. Todd, Primal-dual interior-point methods for self-scaled cones, SIAM Journal on optimization 8 (2) (1998) 324-364.

[45] M. Smith, ABAQUS/Standard User's Manual, Version 6.9, Simulia, 2009.

[46] G. De Saxcé, Z. Q. Feng, The bipotential method: a constructive approach to design the complete contact law with friction and improved numerical algorithms, Mathematical and Computer Modelling 28 (4) (1998) 225-245. doi:10.1016/S0895-7177(98) 00119-8.

[47] T. H. H. Pian, Derivation of element stiffness matrices by assumed stress distributions, AIAA Journal 2 (7) (1964) 1333-1336. doi:10.2514/3.2546.

[48] M. Kempeneers, J.-F. Debongnie, P. Beckers, Pure equilibrium tetrahedral finite elements for global error estimation by dual analysis, International Journal for Numerical Methods in Engineering 81 (4) (2009) 513-536. doi:10.1002/nme.2703.

[49] B. Fraeijs de Veubeke, Displacement and equilibrium models in the finite element method, International Journal for Numerical Methods in Engineering 52 (3) (2001) 287-342. doi:10.1002/nme.339. 
[50] G. Morera, Soluzione generale delle equazioni indefinite dell'equilibrio di un corpo continuo, Atti Accad. Naz. Lincei, Rend. Cl. Fis. Mat. Natur., V. Ser 1 (1) (1892) $137-141$.

[51] J. C. Maxwell, On reciprocal diagrams in space, and their relation to Airy's function of stress, Proceedings of the London Mathematical Society 1 (1) (1866) 58-63.

[52] F. Alizadeh, D. Goldfarb, Second-order cone programming, Mathematical Programming 95 (1) (2003) 3-51. doi:10.1007/s10107-002-0339-5.

[53] Mosek, Mosek modeling manual, User manual, Mosek (2013).

[54] J. Sturm, Using SeDuMi 1.02, a matlab toolbox ofr optimization over symmetric cones, Optimization Methods and Software.

[55] E. Andersen, On formulating quadratic functions in optimization problems, Technical Report TR-1-2013, Mosek (2013).

[56] P. Ladevèze, J. P. Pelle, Mastering calculations in linear and nonlinear mechanics, Mechanical engineering series, Springer Science, New York, 2005, oCLC: ocm55094720.

[57] F. Alauzet, P. Frey, Estimateur d'erreur géométrique et métriques anisotropes pour l'adaptation de maillage. Partie I : aspects théoriques, Institut national de recherche en informatique et en automatique 37 (1) (2006) 55-57. doi:10.1177/ 075910639203700105 .

[58] R. Kučera, J. Machalová, H. Netuka, P. Ženčák, An interior-point algorithm for the minimization arising from $3 \mathrm{~d}$ contact problems with friction, Optimization methods and software 28 (6) (2013) 1195-1217.

[59] J.-F. Debongnie, A general theory of dual error bounds by finite elements, Tech. rep., Université de Liège (1983).

[60] J. Debongnie, H. Zhong, P. Beckers, Dual analysis with general boundary conditions, Computer Methods in Applied Mechanics and Engineering 122 (1-2) (1995) 183-192. doi:10.1016/0045-7825(94)00726-4.

[61] P. Coorevits, P. Hild, M. Hjiaj, A posteriori error control of finite element approximations for Coulomb's frictional contact, SIAM Journal on Scientific Computing 23 (3) (2001) 976-999. doi:10.1137/S1064827500375461.

[62] F. Louf, J.-P. Combe, J.-P. Pelle, Constitutive error estimator for the control of contact problems involving friction, Computers \& Structures 81 (18-19) (2003) 1759-1772. doi:10.1016/S0045-7949(03)00200-1. URL https://linkinghub.elsevier.com/retrieve/pii/S0045794903002001 
[63] F. Ben Belgacem, C. Bernardi, A. Blouza, M. Vohralik, On the unilateral contact between membranes. Part 2: a posteriori analysis and numerical experiments, IMA Journal of Numerical Analysis 32 (3) (2012) 1147-1172. doi:10.1093/imanum/drr003. URL https://academic .oup.com/imajna/article-lookup/doi/10.1093/imanum/ drro03

[64] P. Ladevèze, L. Chamoin, The Constitutive Relation Error Method: A General Verification Tool, in: L. Chamoin, P. Díez (Eds.), Verifying Calculations - Forty Years On: An Overview of Classical Verification Techniques for FEM Simulations, Springer International Publishing, Cham, 2016, pp. 59-94. doi:10.1007/978-3-319-20553-3_4. URL https://doi.org/10.1007/978-3-319-20553-3_4

[65] A. De Coninck, B. De Baets, D. Kourounis, F. Verbosio, O. Schenk, S. Maenhout, J. Fostier, Needles: toward large-scale genomic prediction with marker-byenvironment interaction, Genetics 203 (1) (2016) 543-555. doi:10.1534/genetics. 115.179887.

[66] F. Verbosio, A. D. Coninck, D. Kourounis, O. Schenk, Enhancing the scalability of selected inversion factorization algorithms in genomic prediction, Journal of Computational Science 22 (Supplement C) (2017) $99-108$.

[67] D. Kourounis, A. Fuchs, O. Schenk, Towards the next generation of multiperiod optimal power flow solvers, IEEE Transactions on Power Systems PP (99) (2018) 1-10. doi:10.1109/TPWRS.2017.2789187. 